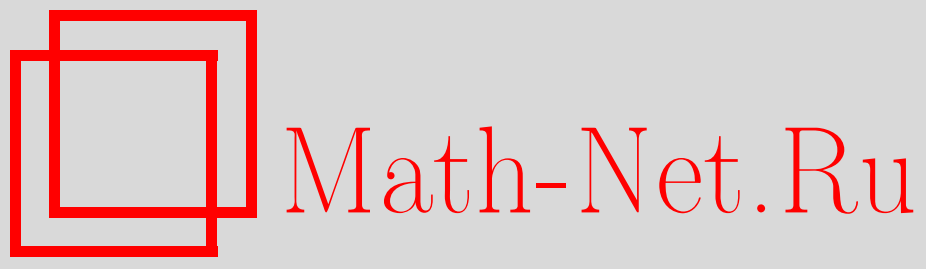

А. А. Обломков, Изоэнергетическая спектральная задача для многомерных разностных операторов, Функи. анализ и его прил., 2002, том 36, выпуск $2,45-61$

DOI: https://doi.org/10.4213/faa190

Использование Общероссийского математического портала MathNet.Ru подразумевает, что вы прочитали и согласны с пользовательским соглашением

http://www. mathnet.ru/rus/agreement

Параметры загрузки:

IP : 54.172 .240 .79

26 апреля 2023 г., 02:26:03

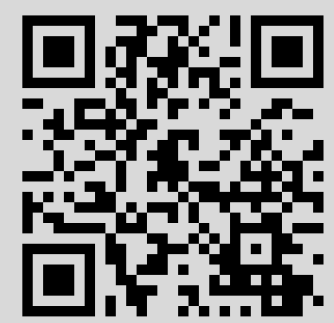




\title{
Изоэнергетическая спектральная задача для многомерных разностных операторов*
}

\author{
(c) 2002. А. А. ОБломков
}

\section{Введение}

Спектральная задача для многомерного уравнения Шрёдингера в случае, когда спектральные данные собираются с одного фиксированного уровня энергии, имеет давнюю историю, связанную с именами Р. Ньютона, П. Сабатье, Б. М. Левитана и др. (см. например книгу Шадана и Сабатье [1], где имеется замечательный исторический обзор с обширным списком литературы).

Алгебро-геометрический анализ этой проблемы был начат Б. А. Дубровиным, И. М. Кричевером и С. П. Новиковым в известной работе [2], где был введен класс двумерных операторов Шрёдингера, конечнозонных по отношению к одному уровню энергии. Собственная функция такого оператора определена на алгебраической кривой и мероморфна вне выделенных точек, в которых она имеет экспоненциальные особенности (так называемая функция Бейкера-Ахиезера). В последующих работах А. П. Веселов и С. П. Новиков нашли достаточные условия на спектральную кривую и дивизор полюсов $\psi$, для которых соответствующий оператор чисто потенциален [3-5].

Исследование разностных операторов с аналогичными свойствами было начато И. М. Кричевером в работах $[6,7]$, где была предложена конструкция для обратной задачи на одном уровне энергии для операторов на двумерной решетке. Прямая задача для операторов на двумерной решетке исследовалась в недавней работе [8], в этой работе также даны ответы на некоторое вопросы, поставленные в работе [7]. Отметим также работу [9], где исследуется обратная спектральная задача для некоторого класса двумерных разностных операторов с ненулевой диагональю.

В работах Т. Каппелера и Д. Бэттига $[10,11]$ была построена компактификация спектрального многообразия для многомерного разностного оператора Шрёдингера при всех уровнях энергии и исследован вопрос об операторах с одинаковыми спектральными многообразиями (непрерывной версии этих результатов посвящены работы $[12,13])$.

В недавних работах С. П. Новикова и И. А. Дынникова $[14,15]$ были найдены некоторые примеры интегрируемых в стиле теории солитонов дискретных операторов на многомерной симплициальной решетке. Эти работы стимулировали дальнейшие исследования в указанном направлении [16], к которому относится и настоящая работа.

\footnotetext{
*Работа поддержана грантом РФФИ № 01-01-00803.
} 
В работе исследуются прямая и обратная спектральные задачи на нулевом уровне энергии для класса операторов $L$, действующих на пространстве $V$ функций, определенных на решетке $\Gamma=\mathbb{Z}^{N}$, по следующей формуле:

$$
(L \psi)(n)=\sum_{\zeta=( \pm 1, \ldots, \pm 1)} a(n ; \zeta) \psi(n+\zeta) .
$$

Предполагается, что коэффициенты оператора удовлетворяют условиям самосопряженности $a(n ; \zeta)=a(n+\zeta ;-\zeta)$ и периодичности $a\left(n+T_{i} e_{i} ; \zeta\right)=a(n ; \zeta)$, $T_{i} \in \mathbb{Z}, i=1, \ldots, N$.

Заметим, что диагональные элементы оператора $L$ равны нулю. В одномерном случае алгебро-геометрический анализ спектральной теории «бездиагональных» операторов $(L \psi)_{n}=c_{n} \psi_{n-1}+c_{n+1} \psi_{n+1}$ был произведен С. П. Новиковым (см. [17]). Спектральными данными в этом случае являлись кривая с симметрией и дивизор на этой кривой. В работе [18] была найдена связь таких операторов с цепочкой Гейзенберга и выявлено различие между четным и нечетным случаями.

Спектральными данными в нашем случае будут наборы гиперповерхностей (спектральные многообразия), оснащенные набором дивизоров, удовлетворяющих некоторым условиям. Первая часть этих условий обобщает соотношения Веселова-Новикова $[3,4]$, вторая часть гарантирует существование многомерных аналогов функции Бейкера-Ахиезера [6]. Спектральные данные обладают замечательным свойством, которое мы называем наличием индуктивной структуры. Это свойство заключается в том, что компоненты, добавляемые при компактификации спектрального многообразия, вместе с ограничениями на них набора дивизоров представляют собой спектральные данные ограничения изначального разностного оператора на некоторую подрешетку. Это позволяет свести задачу восстановления оператора по спектральным данным к одномерному случаю и получить формулы в $\theta$-функциях. Опишем эти спектральные данные и соответствующие соотношения. Аффинной частью спектрального многообразия оператора $L$ называется гиперповерхность $Y=Y_{L}=\left\{\lambda \in\left(\mathbb{C}^{*}\right)^{N} \mid V(\lambda) \cap \operatorname{ker} L \neq 0\right\}$, где $V(\lambda)=\left\{\psi \in V \mid \psi\left(n+T_{i} e_{i}\right)=\lambda_{i} \psi(n)\right\}-$ пространство блоховских функций. Для простоты будем считать, что все $T_{i}$ нечетны (в основной части статьи это ограничение отсутствует). В этом случае для общего $L$ гиперповерхность $Y_{L}$ является неприводимой, инвариантной относительно действия группы $\mathbb{Z}_{2}^{N}$ и меняющей знаки у компонент вектора $\lambda$. Ее фактор по этой группе обозначим через $X=X_{L}$ и будем называть модифицированным спектральным многообразием. Вложение $\left(\mathbb{C}^{*}\right)^{N} \subset\left(\mathbb{C P}^{1}\right)^{N}$ дает естественную компактификацию многообразия $X$. Многообразия, добавляемые при компактификации, имеют аналогичную структуру: $X^{\gamma}\left[\begin{array}{l}i \\ k\end{array}\right]=X_{L^{\gamma}\left[\begin{array}{l}i \\ k\end{array}\right]}$, где оператор $L^{\gamma}\left[\begin{array}{l}i \\ k\end{array}\right], k \in \mathbb{Z}, 1 \leqslant i \leqslant N, \gamma= \pm 1$, является «редукцией» оператора $L$ на $(N-1)$-мерную подрешетку $\Gamma\left[\begin{array}{l}i \\ k\end{array}\right]=\left\{\xi \in \Gamma \mid \xi_{i}=k\right\}$ (точные определения см. в основном тексте). На $X$ естественным образом действует инволюция $\sigma: \lambda \rightarrow \lambda^{-1}$, которая специальным образом переставляет «бесконечности» $X^{\gamma}\left[\begin{array}{l}i \\ k\end{array}\right]$.

Общей точке $\lambda \in Y$ отвечает вектор $\psi(\lambda, n) \in \operatorname{ker} L \cap V(\lambda)$, единственный с точностью до умножения на константу. При этом $\psi(\lambda, 2 n+\varepsilon) / \psi(\lambda, \varepsilon)$, $\varepsilon \in\{0,1\}^{N}$, является корректно определенной функцией на $X$. Таким образом, вектор $\psi(\lambda, 2 n+\varepsilon), n \in \Gamma$, нормированный условием $\psi(\lambda, \varepsilon) \equiv 1$, корректно определен на $X$. Для упрощения формулировок положим $\varepsilon=0$. Тогда существует 
эффективный дивизор $\mathscr{D}_{0}$, не имеющий неприводимых компонент, лежащих на бесконечности (дивизоры с таким свойством будем называть конечными), такой, что выполнены соотношения

$$
\begin{gathered}
\mathscr{D}_{0}+\sigma\left(\mathscr{D}_{0}\right)+\sum_{s=1}^{N} \sum_{p=0}^{\left(T_{s}-1\right) / 2}\left(X^{1}\left[\begin{array}{c}
s \\
2 p+1
\end{array}\right]-X^{-1}\left[\begin{array}{c}
s \\
2 p+1
\end{array}\right]\right)-\sum_{s=1}^{N} X^{+1}\left[\begin{array}{l}
s \\
0
\end{array}\right] \sim \mathscr{K}_{X}, \\
\mathscr{D}_{2 n}:=(\psi(\cdot, 2 n))-\mathscr{D}_{0}+Q\left[\begin{array}{c}
0 \\
2 n
\end{array}\right]-Q\left[\begin{array}{c}
2 n \\
0
\end{array}\right]>0 \quad \forall n \in \Gamma, \\
X^{ \pm 1}\left[\begin{array}{c}
r \\
p+T_{r}
\end{array}\right]=X^{ \pm 1}\left[\begin{array}{c}
r \\
p
\end{array}\right], \quad Q\left[\begin{array}{c}
2 n+2 T_{r} e_{r} \\
2 n
\end{array}\right] \sim 0, \quad r=1, \ldots, N ;
\end{gathered}
$$

здесь $\mathscr{K}_{X}-$ канонический дивизор, а $Q\left[\begin{array}{l}2 n^{1} \\ 2 n^{0}\end{array}\right]=\sum_{s=1}^{N} \sum_{p=n_{s}^{0}}^{n_{s}^{1}}\left(X^{1}\left[\begin{array}{c}s \\ 2 p+1\end{array}\right]-\right.$ $\left.X^{-1}\left[\begin{array}{c}s \\ 2 p+1\end{array}\right]\right)$, где суммирование предполагается лишь в случае $n_{s}^{0} \leqslant n_{s}^{1}$. Соотношение (1) является многомерным дискретным обобщением соотношений ВеселоваНовикова, соотношение (2) обобщает стандартные требования к функции Бейкера-Ахиезера (фиксированная особенность в конечной части и кратности полюсов (нулей) на бесконечности, зависящие от $n$ ), а условие (3) гарантирует квазипериодичность функции $\psi$. При $N=2$ соотношение $(2)$ было выписано в работе И. М. Кричевера [6], а соотношение (1) - в работе автора [8].

Многообразие $X$ вместе с дивизором $\mathscr{D}_{0}$ являются спектральными данными нашей задачи. Тот факт, что набор $\left\{X^{\gamma}\left[\begin{array}{l}i \\ k\end{array}\right], \mathscr{D} \cap X^{\gamma}\left[\begin{array}{l}i \\ k\end{array}\right]\right\}$ является набором спектральных данных прямой задачи для операторов $L^{\gamma}\left[\begin{array}{l}i \\ k\end{array}\right]$, является для нас ключевым. Он позволяет свести решение обратной спектральной задачи для $N$-мерного оператора к решению спектральных задач для двумерных операторов, а в двумерном случае эта задача решена в [7] (см. также [8]) и имеются явные $\theta$-функциональные формулы.

На пространстве операторов $L$ действует калибровочная группа по формуле $a^{\prime}(n ; \zeta)=g(n) g(n+\zeta) a(n ; \zeta)$, где $g$ - произвольная периодическая функция на решетке. Нетрудно видеть, что она сохраняет спектральные данные, отвечающие нулевому уровню энергии. Основным результатом работы является утверждение о том, что спектральные данные $\left\{X_{L}, \mathscr{D}_{0}\right\}$ однозначно определяют калибровочный класс оператора $L$. Оператор $L$ может быть восстановлен в терминах $\theta$-функций некоторых кривых, лежащих на «бесконечности» $X_{L}$. Например, при $N=3$ обратная спектральная задача решается следующим образом. Определим $\mathscr{D}_{2 n}$ по $\mathscr{D}_{0}$ формулой $(2)$, а затем по спектральным данным $\left\{X^{\gamma}\left[\begin{array}{c}i \\ 2 n_{i}-\gamma\end{array}\right], \mathscr{D}_{2 n} \cap X^{\gamma}\left[\begin{array}{c}i \\ 2 n_{i}-\gamma\end{array}\right]\right\}$ восстановим двумерный оператор $L^{\gamma}\left[\begin{array}{c}i \\ 2 n_{i}\end{array}\right]$ по схеме работы [6]. Коэффициенты $L^{\gamma}\left[\begin{array}{c}i \\ 2 s\end{array}\right], i, s \in \mathbb{Z}, \gamma= \pm 1$, дают нам все коэффициенты оператора $L$.

Автор благодарен проф. А. П. Веселову за постановку задачи в двумерном случае, полезные обсуждения и предложения по улучшению первоначального текста.

\section{§1. Геометрия $N$-мерной кубической решетки, редуцированные операторы и их спектральные многообразия}

Здесь и далее используются следующие соглашения. Пусть $\mu=\left(\mu_{1}, \ldots, \mu_{N}\right)$, $k=\left(k_{1}, \ldots, k_{N}\right) \in \mathbb{Z}^{N}, l=\left(l_{1}, \ldots, l_{r}\right) \in \mathbb{Z}^{r}, l_{i} \leqslant N$; тогда $\mu_{l}=\left(\mu_{l_{1}}, \ldots, \mu_{l_{r}}\right)$ и $\mu^{k}=\mu_{1}^{k_{1}} \cdots \mu_{N}^{k_{N}}$. 
Для описания алгебраической структуры спектральных данных оператора $L$ нам понадобятся связанные с ним операторы $L_{j}^{\gamma}\left[\begin{array}{l}i \\ k\end{array}\right]$, действующие в пространстве $V^{M}$ функций на решетке $\Gamma^{M}=\mathbb{Z}^{N}, M \leqslant N$. Здесь и далее $i \in \mathbb{Z}^{N-M}$ и $j \in \mathbb{Z}^{M}$ задают разбиение множества $\{1, \ldots, N\}$ на два непересекающихся подмножества: $\left\{i_{1}, \ldots, i_{N-M}\right\} \cup\left\{j_{1}, \ldots, j_{M}\right\}=\{1, \ldots, N\}, k \in \mathbb{Z}^{N-M}, \gamma \in\{+1,-1\}^{N-M}$. Действие оператора $L_{j}^{\gamma}\left[\begin{array}{l}i \\ k\end{array}\right]$ задается формулой

$$
\left(L_{j}^{\gamma}\left[\begin{array}{l}
i \\
k
\end{array}\right] \psi\right)(m)=\sum_{\zeta, \zeta_{i}=\gamma} a(n ; \zeta) \psi\left(m+\sum_{r=1}^{M} \zeta_{j_{r}} e_{r}\right),
$$

где $\zeta=( \pm 1, \ldots, \pm 1), m \in \Gamma^{M}=\mathbb{Z}^{M}, n_{i}=k, n_{j}=m$. Вектор $j$ фиксирует порядок переменных, и когда нам будет неважен этот порядок, мы будем индекс $j$ опускать. Отметим, что оператор $L$ является частным случаем оператора $L_{j}^{\gamma}\left[\begin{array}{l}i \\ k\end{array}\right]$ (при $M=N, \gamma, i=\varnothing, j=(1, \ldots, N)$ ).

Поясним неформально смысл оператора $L^{\gamma}\left[\begin{array}{l}i \\ k\end{array}\right]$. Пусть $M=N-1, \gamma= \pm 1, i=$ $N$; тогда $(L \psi)(n)=\left(L^{+1}\left[\begin{array}{c}N \\ n_{N}\end{array}\right] \varphi^{+1}\right)(\tilde{n})+\left(L^{-1}\left[\begin{array}{c}N \\ n_{N}\end{array}\right] \varphi^{-1}\right)(\tilde{n})$, где $\tilde{n}=\left(n_{1}, \ldots, n_{N-1}\right)$, $\varphi^{\gamma}(m)=\psi\left(m_{1}, \ldots, m_{N-1}, n_{N}+\gamma\right)$. То есть «локально» оператор $L$ представим в виде суммы $L^{+1}\left[\begin{array}{c}N \\ n_{N}\end{array}\right] \oplus L^{-1}\left[\begin{array}{c}N \\ n_{N}\end{array}\right]$. Оператор $L^{\gamma}\left[\begin{array}{l}i \\ k\end{array}\right]$ можно рассматривать как редукцию оператора $L$ на подрешетку $\Gamma\left[\begin{array}{l}i \\ k\end{array}\right]=\left\{\xi \in \Gamma^{N} \mid \xi_{i}=k\right\}$ в направлении $\gamma$. Точки $n+\zeta, \zeta=( \pm 1, \ldots, \pm 1)$ являются вершинами $N$-мерного куба. На оператор $L^{\gamma}\left[\begin{array}{l}i \\ k\end{array}\right]$ можно смотреть как на оператор, действующий на пространстве $\left\{\psi \in V^{N}\right.$ $\left.\operatorname{supp} \psi \subset \Gamma\left[\begin{array}{c}i \\ k+\gamma\end{array}\right]\right\} \cong V^{M}$. Следуя идеологии работы [14], будем считать, что в каждую вершину $n$ входят ребра, соединяющие эту вершину с вершинами $n+\zeta$, и на этих ребрах написаны числа $a(n ; \zeta)$. Для вычисления $\left(L^{\gamma}\left[\begin{array}{l}i \\ k\end{array}\right] \psi\right)\left(n_{j}\right)$ необходимо взять соответствующую грань куба, т.е. вершины $n+\zeta$, такие, что $n_{i}=k, \zeta_{i}=\gamma$, и просуммировать значения $\psi$ по вершинам этой грани с весами $a(n ; \xi)$, написанными на соответствующих ребрах.

Пространство $V^{M}(\lambda)=\left\{\psi \in V^{M} \mid \psi\left(n+T_{r} e_{r}\right)=\lambda_{r} \psi(n), r=1, \ldots, M\right\}$ будем называть пространством блоховских функций с множителем Флоке $\lambda$. Рассмотрим ограничение оператора $L^{\gamma}\left[\begin{array}{l}i \\ k\end{array}\right]$ на подпространство $V^{M}(\lambda)$ и обозначим его символом $L^{\gamma}\left[\begin{array}{l}i \\ k\end{array}\right]_{\lambda}$. Множество точек $Y^{\mathrm{aff}, \gamma}\left[\begin{array}{l}i \\ k\end{array}\right]=\left\{\lambda \in\left(\mathbb{C}^{*}\right)^{M} \mid \operatorname{ker} L^{\gamma}\left[\begin{array}{l}i \\ k\end{array}\right]_{\lambda} \neq 0\right\}$ будем называть аффинной частью спектрального многообразия оператора $L^{\gamma}\left[\begin{array}{l}i \\ k\end{array}\right]$. Выбрав естественную в этом случае компактификацию многообразия $\left(\mathbb{C}^{*}\right)^{M} \subset$ $\left(\mathbb{C P}^{1}\right)^{M}$, получаем компактификацию $Y^{\gamma}\left[\begin{array}{l}i \\ k\end{array}\right]$ многообразия $Y^{\mathrm{aff}, \gamma}\left[\begin{array}{l}i \\ k\end{array}\right]$. Множество $Y^{\gamma}\left[\begin{array}{l}i \\ k\end{array}\right] \backslash Y^{\mathrm{aff}, \gamma}\left[\begin{array}{l}i \\ k\end{array}\right]$ будем обозначать символом $Y^{\infty, \gamma}\left[\begin{array}{l}i \\ k\end{array}\right]$.

Полученное многообразие $Y^{\gamma}\left[\begin{array}{l}i \\ k\end{array}\right]$, вообще говоря, приводимо, и чтобы описать неприводимые компоненты этого многообразия, нам понадобится следующая конструкция. Пространство $V^{M}$ естественным образом разлагается в сумму: $V^{M}=$ $\bigoplus_{\varepsilon} V_{\varepsilon}^{M}, V_{\varepsilon}^{M}=\left\{\psi \in V^{M} \mid \operatorname{supp} \psi \subset \Gamma_{\varepsilon}^{M}\right\}$, где $\varepsilon \in \mathbb{Z}_{2}^{M}, \Gamma_{\varepsilon}^{M}=\left\{\xi \in \Gamma^{M} \mid \xi=\varepsilon\right.$ $(\bmod 2)\}$. Это разложение не может быть перенесено на пространство блоховских функций, так как сдвиг на вектор, имеющий хотя бы одну нечетную координату, перемешивает узлы разных типов. Но эту сложность можно обойти с помощью следующей конструкции. Пусть $T_{r}^{\prime}=T_{r} / 2$, если $T_{r}$ четно, и $T_{r}^{\prime}=T_{r}$ в противном случае. Введем пространства $W_{\varepsilon}^{M}(\mu)=\left\{\psi \in V_{\varepsilon}^{M} \mid \psi\left(m+2 T_{j_{r}}^{\prime} e_{r}\right)=\mu_{r} \psi(m), r=\right.$ 
$1, \ldots, M\}$ и отображения $\Phi: \mathbb{C}^{M} \rightarrow \mathbb{C}^{M}, \Phi(\lambda)=\mu$, где $\mu_{r}=\lambda_{r}^{2}$, если $T_{j_{r}}$ нечетно, и $\mu_{r}=\lambda_{r}$ в противном случае. Введем обозначения для четных и нечетных периодов: $l \in \mathbb{Z}^{S}, h \in \mathbb{Z}^{M-S}, S \leqslant M,\left\{l_{1}, \ldots, l_{S}\right\} \cup\left\{h_{1}, \ldots, h_{M-S}\right\}=\{1, \ldots, M\}$; если $T_{j_{r}}$ четно, то $r \in\left\{l_{1}, \ldots, l_{S}\right\}$, в противном случае $r \in\left\{h_{1}, \ldots, h_{M-S}\right\}$. Определим отображение $F_{\varepsilon}^{\lambda}: W_{\varepsilon}^{M}(\mu) \rightarrow V^{M}(\lambda)$, где $\mu=\Phi(\lambda)$, следующим образом: $F_{\varepsilon}^{\lambda}(\varphi)=\psi$, причем если $m_{l}=\varepsilon_{l}(\bmod 2)$, то $\psi(m)=\varphi\left(m-\sum_{r=1}^{M} \rho_{r} T_{r} e_{r}\right) \mu^{\rho}$, где $\rho_{r}=1$, когда $T_{j_{r}}$ нечетно и $m_{r} \neq \varepsilon_{r}(\bmod 2)$, и $\rho_{r}=0$ в противном случае, а если $m_{l} \neq \varepsilon_{l}(\bmod 2)$, то $\psi(m)=0$.

Символом $L_{\varepsilon}^{\gamma}\left[\begin{array}{l}i \\ k\end{array}\right]_{\mu}$ будем обозначать ограничение оператора $L^{\gamma}\left[\begin{array}{l}i \\ k\end{array}\right]$ на пространство $W_{1-\varepsilon}^{M}(\mu), L_{\varepsilon}^{\gamma}\left[\begin{array}{l}i \\ k\end{array}\right]_{\mu}: W_{1-\varepsilon}^{M}(\mu) \rightarrow W_{\varepsilon}^{M}(\mu)$. Нетрудно видеть, что $F_{\varepsilon}^{\lambda}$ корректно определено и инъективно. При этом имеем $F_{1-\varepsilon}^{\lambda}\left(\operatorname{ker} L_{\varepsilon}^{\gamma}\left[\begin{array}{l}i \\ k\end{array}\right]_{\mu}\right) \subset \operatorname{ker} L^{\gamma}\left[\begin{array}{l}i \\ k\end{array}\right]_{\lambda}$, $\mu=\Phi(\lambda)$. Пусть $X_{\varepsilon}^{\mathrm{aff}, \gamma}\left[\begin{array}{l}i \\ k\end{array}\right]=\left\{\mu \in\left(\mathbb{C}^{*}\right)^{M} \mid \operatorname{ker} L_{\varepsilon}^{\gamma}\left[\begin{array}{l}i \\ k\end{array}\right]_{\mu} \neq 0\right\}, X_{\varepsilon}^{\gamma}\left[\begin{array}{l}i \\ k\end{array}\right]=\overline{X_{\varepsilon}^{\mathrm{aff}, \gamma}\left[\begin{array}{l}i \\ k\end{array}\right]} \subset$ $\left(\mathbb{C P}^{1}\right)^{M}, Y_{\varepsilon}^{\gamma}\left[\begin{array}{l}i \\ k\end{array}\right]=\Phi^{-1}\left(X_{\varepsilon}^{\gamma}\left[\begin{array}{l}i \\ k\end{array}\right]\right) \subset Y^{\gamma}\left[\begin{array}{l}i \\ k\end{array}\right]$. Добавляемую при компактификации бесконечную часть многообразия $X_{\varepsilon}^{\gamma}\left[\begin{array}{l}i \\ k\end{array}\right]$ будем обозначать $X_{\varepsilon}^{\infty, \gamma}\left[\begin{array}{l}i \\ k\end{array}\right]=X_{\varepsilon}^{\gamma}\left[\begin{array}{l}i \\ k\end{array}\right] \backslash$ $X_{\varepsilon}^{\mathrm{aff}, \gamma}\left[\begin{array}{l}i \\ k\end{array}\right]$. Символом $V_{\varepsilon}^{M}(\lambda) \subset V^{M}(\lambda)$ обозначим $F_{\varepsilon}^{\lambda}\left(W_{\varepsilon}^{M}(\mu)\right)$. Так как сдвиг на вектор $T_{j_{h_{r}}} e_{h_{r}}, r=1, \ldots, M-S$, переводит пространство $W_{1-\varepsilon}(\mu)$ в пространство $W_{1-\varepsilon^{\prime}}(\mu), \varepsilon^{\prime}=\varepsilon+e_{h_{r}}(\bmod 2)$, и оставляет инвариантным оператор $L^{\gamma}\left[\begin{array}{l}i \\ k\end{array}\right]$, то $V_{1-\varepsilon}(\lambda)=V_{1-\varepsilon^{\prime}}(\lambda), F_{1-\varepsilon}^{\lambda}\left(\operatorname{ker} L_{\varepsilon}^{\gamma}\left[\begin{array}{l}i \\ k\end{array}\right]_{\mu}\right)=F_{1-\varepsilon^{\prime}}^{\lambda}\left(\operatorname{ker} L_{\varepsilon^{\prime}}^{\gamma}\left[\begin{array}{l}i \\ k\end{array}\right]_{\mu}\right) ;$ следовательно, $Y_{\varepsilon}^{\gamma}\left[\begin{array}{l}i \\ k\end{array}\right]=Y_{\varepsilon^{\prime}}^{\gamma}\left[\begin{array}{l}i \\ k\end{array}\right]$, если $\varepsilon_{l}=\varepsilon_{l}^{\prime}$. Подсчетом размерностей можно показать, что имеет место разложение $V(\lambda)=\bigoplus_{\varepsilon, \varepsilon_{h}=0} V_{\varepsilon}(\lambda)$. Так как $F_{1-\varepsilon}^{\lambda}\left(\operatorname{ker} L_{\varepsilon}^{\gamma}\left[\begin{array}{l}i \\ k\end{array}\right]_{\mu}\right)=V_{\varepsilon}^{M}(\lambda) \cap$ $\operatorname{ker} L^{\gamma}\left[\begin{array}{l}i \\ k\end{array}\right]$, то $Y^{\gamma}\left[\begin{array}{l}i \\ k\end{array}\right]=\bigcup_{\varepsilon, \varepsilon_{h}=0} Y_{\varepsilon}^{\gamma}\left[\begin{array}{l}i \\ k\end{array}\right]$. Пусть $X^{\gamma}\left[\begin{array}{l}i \\ k\end{array}\right]=\Phi\left(Y^{\gamma}\left[\begin{array}{l}i \\ k\end{array}\right]\right)=\bigcup_{\varepsilon, \varepsilon_{h}=0} X_{\varepsilon}^{\gamma}\left[\begin{array}{l}i \\ k\end{array}\right]$. Многообразие $X^{\gamma}\left[\begin{array}{l}i \\ k\end{array}\right]\left(X_{\varepsilon}^{\gamma}\left[\begin{array}{l}i \\ k\end{array}\right]\right)$ будем называть модифицзированным спектральным многообразием оператора $L^{\gamma}\left[\begin{array}{l}i \\ k\end{array}\right]$ ( $\left.L_{\varepsilon}^{\gamma}\left[\begin{array}{l}i \\ k\end{array}\right]\right)$.

Наиболее просто приведенная выше конструкция выглядит в случае, когда все $T_{j_{r}}$ четны. Тогда $\mu=\lambda, W_{\varepsilon}(\mu)=V_{\varepsilon}(\lambda), V(\lambda)=\bigoplus_{\varepsilon} V_{\varepsilon}(\lambda), X_{\varepsilon}^{\gamma}\left[\begin{array}{l}i \\ k\end{array}\right]=Y_{\varepsilon}^{\gamma}\left[\begin{array}{l}i \\ k\end{array}\right]$ и задача нахождения $\operatorname{ker} L^{\gamma}\left[\begin{array}{l}i \\ k\end{array}\right]_{\lambda}$ эквивалентна нахождению $\operatorname{ker} L_{\varepsilon}^{\gamma}\left[\begin{array}{l}i \\ k\end{array}\right]_{\lambda}$ для всех $\varepsilon$. В случае же общего $T_{j}$ приведенная выше конструкция является сведением к четному случаю. То есть мы рассматриваем $L^{\gamma}\left[\begin{array}{l}i \\ k\end{array}\right]$ не как $T_{j}$-периодический оператор, а как $2 T_{j}^{\prime}$-периодический оператор. Соответственно множители Флоке $\lambda$ заменяются на множители Флоке $\mu$. Но следует помнить, что в действительности коэффициенты оператора $L^{\gamma}\left[\begin{array}{l}i \\ k\end{array}\right] \quad T_{j}$-периодичны, из чего следует, что

$$
X_{\varepsilon}^{\gamma}\left[\begin{array}{l}
i \\
k
\end{array}\right]=X_{\varepsilon^{\prime}}^{\gamma}\left[\begin{array}{l}
i \\
k
\end{array}\right]=X_{\varepsilon}^{\gamma}\left[\begin{array}{c}
i \\
k+T_{i_{r}} e_{r}
\end{array}\right], \quad r=1, \ldots, N-M
$$

где $\varepsilon_{l}=\varepsilon_{l}^{\prime}$. Необходимость такого сведения мотивирована тем, что $\operatorname{det} L^{\gamma}\left[\begin{array}{l}i \\ k\end{array}\right]_{\lambda}$ является рациональной функцией от $\lambda_{h_{r}}^{2}, r=1, \ldots, M-S$, и переход от $Y^{\gamma}\left[\begin{array}{l}i \\ k\end{array}\right] \mathrm{K}$ $X^{\gamma}\left[\begin{array}{l}i \\ k\end{array}\right]$ отвечает факторизации многообразия $Y^{\gamma}\left[\begin{array}{l}i \\ k\end{array}\right]$ по инволюциям $\lambda_{h_{r}} \rightarrow-\lambda_{h_{r}}$, $r=1, \ldots, M-S$.

Открытое по Зарисскому множество операторов $L$, таких, что $X_{\varepsilon}^{\gamma}\left[\begin{array}{l}i \\ k\end{array}\right]$ для всех $\varepsilon$ являются неприводимыми, обозначим символом $U_{i r}^{\gamma}\left[\begin{array}{l}i \\ k\end{array}\right]$. Непустота этого множества будет доказана в лемме 4. 


\section{§2. Спектральные данные: индуктивная структура}

Наличие индуктивной структуры заключается в возможности сведения изучения одного оператора $L$, действующего в размерности $N$, к изучению большого числа операторов $L_{j}^{\gamma}\left[\begin{array}{l}i \\ k\end{array}\right]$ такого же типа, действующих уже в размерности $M<N$.

Выберем базис $\left\{e_{\varepsilon, j}^{M}(\mu)\right\}$ в $W_{\varepsilon}^{M}(\mu)$, такой, что матрица оператора $L^{\gamma}\left[\begin{array}{l}i \\ k\end{array}\right]$, записанная в этом базисе, имеет блочную структуру, отражающую индуктивную структуру оператора $L^{\gamma}\left[\begin{array}{l}i \\ k\end{array}\right]$. Пусть $0 \leqslant m_{r}^{\prime}<T_{j_{r}}^{\prime}, a=1+\sum_{b=1}^{M} m_{b}^{\prime} \prod_{r=b+1}^{M} T_{j_{r}}^{\prime}$; тогда $e_{\varepsilon, j, a}^{M}(\mu)$ однозначно определяется из условия $e_{\varepsilon, j, a}(\mu) \in W_{\varepsilon}^{M}(\mu), e_{\varepsilon, j, a}(\mu, m)=$ $\prod_{b=1}^{M} \delta_{\varepsilon_{b}+2 m_{b}^{\prime}}^{m_{b}}$ при $0 \leqslant m-\varepsilon<2 T_{j}^{\prime}$. Матрицу оператора $L_{\varepsilon}^{\gamma}\left[\begin{array}{l}i \\ k\end{array}\right]_{\mu}$, записанную в базисах $\left\{e_{1-\varepsilon, j}(\mu)\right\},\left\{e_{\varepsilon, j}(\mu)\right\}$, будем обозначать символом $\mathbf{M}_{\varepsilon, j}^{\gamma}\left[\begin{array}{l}i \\ k\end{array}\right](\mu)$. Эта матрица имеет блочно-двудиагональную структуру с блоками вида $\mathbf{M}_{\tilde{\varepsilon}, \tilde{j}}^{\gamma, \pm 1}\left[\begin{array}{c}i, t \\ k, u\end{array}\right]$ :

$$
\mathbf{M}_{\varepsilon, j}^{\gamma}\left[\begin{array}{c}
i \\
k
\end{array}\right]=\left|\begin{array}{cccc}
\mathbf{M}_{\tilde{\varepsilon}, \tilde{j}}^{\gamma^{\prime}}\left[\begin{array}{c}
i, j_{1} \\
k, 0
\end{array}\right] & 0 & \ldots & \mathbf{M}_{\tilde{\varepsilon}, \tilde{j}}^{\gamma^{\prime \prime}}\left[\begin{array}{c}
i, j_{1} \\
k, 0
\end{array}\right] \mu_{1}^{-1} \\
\mathbf{M}_{\tilde{\varepsilon}, \tilde{j}}^{\gamma^{\prime \prime}}\left[\begin{array}{c}
i, j, 2 \\
k, 2
\end{array}\right] & \mathbf{M}_{\tilde{\varepsilon}, \tilde{j}}^{\gamma^{\prime}}\left[\begin{array}{c}
i, j_{1} \\
k, 2
\end{array}\right] & \ldots & 0 \\
\vdots & \vdots & \ddots & \vdots \\
0 & 0 & \ldots & \mathbf{M}_{\tilde{\varepsilon}, \tilde{j}}^{\gamma^{\prime}}\left[\begin{array}{c}
i, j_{1} \\
k, 2\left(T_{j_{1}}^{\prime}-1\right)
\end{array}\right]
\end{array}\right|
$$

при $\varepsilon_{j_{1}}=1$,

$$
\mathbf{M}_{\varepsilon, j}^{\gamma}\left[\begin{array}{c}
i \\
k
\end{array}\right]=\left|\begin{array}{cccc}
\mathbf{M}_{\tilde{\varepsilon}, \tilde{j}}^{\gamma^{\prime \prime}}\left[\begin{array}{c}
i, j_{1} \\
k, 1
\end{array}\right] & \mathbf{M}_{\tilde{\varepsilon}, \tilde{j}}^{\gamma^{\prime}}\left[\begin{array}{c}
i, j_{1} \\
k, 1
\end{array}\right] & \ldots & 0 \\
0 & \mathbf{M}_{\tilde{\varepsilon}, \tilde{j}}^{\gamma^{\prime \prime}}\left[\begin{array}{c}
i, j_{1} \\
k, 3
\end{array}\right] & \ldots & 0 \\
\vdots & \vdots & \ddots & \vdots \\
\mathbf{M}_{\tilde{\varepsilon}, \tilde{j}}^{\gamma^{\prime}\left[\begin{array}{c}
i, j_{1} \\
k, 2 T_{j_{1}}^{\prime}-1
\end{array}\right] \mu_{1}} & 0 & \ldots & \mathbf{M}_{\tilde{\varepsilon}, \tilde{j}}^{\gamma^{\prime \prime}}\left[\begin{array}{c}
i, j_{1} \\
k, 2 T_{j_{1}}^{\prime}-1
\end{array}\right]
\end{array}\right|
$$

при $\varepsilon_{j_{1}}=0$; здесь использованы обозначения $\tilde{j}=\left(j_{2}, \ldots, j_{M}\right), \tilde{\varepsilon}=\left(\varepsilon_{2}, \ldots, \varepsilon_{M}\right)$, $\gamma^{\prime}=(\gamma, 1), \gamma^{\prime \prime}=(\gamma,-1)$. Индукцией по $M$ можно показать, что

$$
\left(\mathbf{M}_{\varepsilon, j}^{\gamma}\left[\begin{array}{l}
i \\
k
\end{array}\right](\mu)\right)^{t}=\mathbf{M}_{1-\varepsilon, j}^{-\gamma}\left[\begin{array}{c}
i \\
k+\gamma
\end{array}\right]\left(\mu^{-1}\right),
$$

где $\mu^{-1}=\left(\mu_{1}^{-1}, \ldots, \mu_{M}^{-1}\right)$, а символ $t$ означает транспонирование.

Уравнение $R_{\varepsilon, j}^{\gamma}\left[\begin{array}{l}i \\ k\end{array}\right](\mu)=\operatorname{det}\left(M_{\varepsilon, j}^{\gamma}\left[\begin{array}{l}i \\ k\end{array}\right](\mu)\right)=0$ определяет многообразие $X_{\varepsilon}^{\gamma}\left[\begin{array}{l}i \\ k\end{array}\right]$. Нетрудно видеть, что $R_{\varepsilon, j}^{\gamma}\left[\begin{array}{l}i \\ k\end{array}\right](\mu)=C R_{\varepsilon, \tau(j)}^{\gamma}\left[\begin{array}{l}i \\ k\end{array}\right](\tau(\mu))$, где $C \neq 0-$ константа, $\tau \in$ $S_{M}$ - перестановка, $\tau(j)=\left(j_{\tau(1)}, \ldots, j_{\tau(M)}\right), \tau(\mu)=\left(\mu_{\tau(1)}, \ldots, \mu_{\tau(M)}\right)$; поэтому мы будем опускать $j$ в обозначении $R_{\varepsilon, j}^{\gamma}\left[\begin{array}{l}i \\ k\end{array}\right]$. Из вида матрицы $M_{\varepsilon, j}^{\gamma}\left[\begin{array}{l}i \\ k\end{array}\right]$ следует, что $R_{\varepsilon, j}^{\gamma}\left[\begin{array}{l}i \\ k\end{array}\right]$ является полиномом от $\mu_{1}^{\left(1-2 \varepsilon_{j_{1}}\right)}$ степени $\prod_{t=2}^{M} T_{j_{t}}^{\prime}$, и из предыдущего рассуждения также следует, что $R_{\varepsilon}^{\gamma}\left[\begin{array}{l}i \\ k\end{array}\right]$ является полиномом от $\mu_{r}^{\left(1-2 \varepsilon_{j_{r}}\right)}$ степени $\left(T_{j_{r}}^{\prime}\right)^{-1} \prod_{p=1}^{M} T_{j_{p}}^{\prime}, r=1, \ldots, M$. Суммируем полученные нами наблюдения:

Лемма 1. Для общего оператора L справедливы следующие утверждения: $R_{\varepsilon}^{\gamma}\left[\begin{array}{l}i \\ k\end{array}\right](\mu)$ является полиномом по $\mu_{r}^{\left(1-2 \varepsilon_{j_{r}}\right)} u \operatorname{deg}_{\mu_{r}}^{\left(1-2 \varepsilon_{j_{r}}\right)} R_{\varepsilon}^{\gamma}\left[\begin{array}{l}i \\ k\end{array}\right]=\left(T_{j_{r}}^{\prime}\right)^{-1} \prod_{t=1}^{M} T_{j_{t}}^{\prime}$;

$$
R_{\varepsilon}^{\gamma}\left[\begin{array}{l}
i \\
k
\end{array}\right]=R_{\varepsilon^{\prime}}^{\gamma}\left[\begin{array}{c}
i \\
k+T_{i_{r}}^{\prime}
\end{array}\right]
$$


где $r=1, \ldots, N-M, \varepsilon_{l}=\varepsilon_{l}^{\prime}$;

$$
\begin{gathered}
R_{\varepsilon}^{\gamma}\left[\begin{array}{l}
i \\
k
\end{array}\right](\mu)=R_{1-\varepsilon}^{-\gamma}\left[\begin{array}{c}
i \\
k+\gamma
\end{array}\right]\left(\mu^{-1}\right) \\
\left.R_{\varepsilon}^{\gamma}\left[\begin{array}{l}
i \\
k
\end{array}\right](\mu) \mu_{p}^{T_{j_{p}}^{\prime} \delta_{2 \varepsilon_{p}-1}^{w}}\right|_{\mu_{p}^{-w}=0}=\prod_{r=0}^{T_{j_{p}}^{\prime}-1} R_{\tilde{\varepsilon}}^{\gamma^{\prime}\left[\begin{array}{c}
i, j_{r} \\
k, 1-\varepsilon_{p}+2 r
\end{array}\right](\tilde{\mu}),}
\end{gathered}
$$

где $p=1, \ldots, M, w= \pm 1, \gamma^{\prime}=(\gamma, w), \tilde{\mu}=\left(\mu_{1}, \ldots, \mu_{p-1}, \mu_{p+1}, \ldots, \mu_{M}\right), \tilde{\varepsilon}=$ $\left(\varepsilon_{1}, \ldots, \varepsilon_{p-1}, \varepsilon_{p+1}, \ldots, \varepsilon_{M}\right)$.

Формула (7) является непосредственным следствием блочно-двудиагональной структуры матрицы $\mathbf{M}_{\varepsilon}^{\gamma}\left[\begin{array}{l}i \\ k\end{array}\right]$.

Из соотношения (6) следует, что естественная инволюция $\sigma$ на $\left(\mathbb{C P}^{1}\right)^{N}, \sigma(\mu)=$ $\mu^{-1}$, действует на $X_{\varepsilon}^{\gamma}\left[\begin{array}{l}t \\ u\end{array}\right]$ по следующему правилу:

$$
\sigma\left(X_{\varepsilon}^{\gamma}\left[\begin{array}{l}
i \\
k
\end{array}\right]\right)=X_{1-\varepsilon}^{-\gamma}\left[\begin{array}{c}
i \\
k+\gamma
\end{array}\right] \text {. }
$$

Наличие этой инволюции является следствием самосопряженности оператора $L^{\gamma}\left[\begin{array}{l}i \\ k\end{array}\right]$.

Символом $\left(\mathbb{C P}^{1}\right)_{\mu}^{M}$ будем обозначать многообразие $\left(\mathbb{C P}^{1}\right)^{M}$ с фиксированным набором координат $\mu$ : $\mu_{r}$ является координатой на $r$-м $\mathbb{C P}^{1}$. Теперь введем определение набора многообразий, совместимого с группой периодов $T$. Набор $\left\{X_{\varepsilon} \subset\left(\mathbb{C P}^{1}\right)_{\mu}^{N}\right\}$, где $X_{\varepsilon} \subset\left(\mathbb{C P}^{1}\right)^{N}-$ неприводимые гладкие гиперповерхности, является набором многообразий, совместимых с группой периодов $T$, если

- $X_{\varepsilon}-$ многообразие степени $\left(\left(T_{1}^{\prime}\right)^{-1}, \ldots,\left(T_{N}^{\prime}\right)^{-1}\right) \prod_{u=1}^{N} T_{u}^{\prime}$;

- $X_{\varepsilon} \cap\left\{\mu_{i_{1}}^{-\gamma_{1}}=0, \ldots, \mu_{i_{N-M}}^{-\gamma_{N-M}}=0\right\}=\bigcup_{0 \leqslant k<T_{i}^{\prime}} X_{\varepsilon_{j}}^{\gamma}\left[\begin{array}{c}i \\ 2 k+\varepsilon_{i}\end{array}\right]$, где $X_{\varepsilon_{j}}^{\gamma}\left[{ }^{i}.\right]-$ неприводимое гладкое многообразие степени $\left(\left(T_{j_{1}}^{\prime}\right)^{-1}, \ldots,\left(T_{j_{M}}^{\prime}\right)^{-1}\right) \prod_{u=1}^{N-M} T_{j_{u}}^{\prime}$;

- выполнены соотношения (4), (8).

Обозначим символом $U_{s m}^{\gamma}\left[\begin{array}{l}i \\ k\end{array}\right]$ множество операторов $L$, таких, что $X_{\varepsilon}^{\gamma}\left[\begin{array}{l}i \\ k\end{array}\right]$ неособо для всех $\varepsilon$. Это множество открыто по Зарисскому и, как будет доказано в лемме 4, непусто. Из леммы 1 следует, что для $L \in \bigcap_{i, k, \gamma} U_{s m}^{\gamma}\left[\begin{array}{l}i \\ k\end{array}\right] \cap U_{i r}^{\gamma}\left[\begin{array}{l}i \\ k\end{array}\right]$ набор $\left\{X_{\varepsilon}\right\}$ совместим с набором периодов $T$, где $X_{\varepsilon}$ есть набор спектральных многообразий оператора $L$, т. е. $X_{\varepsilon}^{\varnothing}\left[\begin{array}{l}\varnothing \\ \varnothing\end{array}\right]$.

Заметим, что из данного определения следует, что $\left\{X_{\varepsilon_{j}}^{\gamma}\left[\begin{array}{l}i \\ k\end{array}\right]\right\}$ является многообразием, совместимым с набором периодов $T_{j}$, и

$$
X_{\varepsilon_{j}}^{\gamma}\left[\begin{array}{l}
i \\
k
\end{array}\right] \cap X_{\varepsilon_{j^{\prime}}}^{\gamma^{\prime}}\left[\begin{array}{l}
i^{\prime} \\
k^{\prime}
\end{array}\right]=X_{\varepsilon_{\tilde{j}}}^{\gamma, \gamma^{\prime}}\left[\begin{array}{c}
i, i^{\prime} \\
k, k^{\prime}
\end{array}\right]
$$

при $\left\{i_{1}, \ldots, i_{M}\right\} \cap\left\{i_{1}^{\prime}, \ldots, i_{M^{\prime}}^{\prime}\right\}=\varnothing, \tilde{j}=\left\{j_{1}, \ldots, j_{M}\right\} \cap\left\{j_{1}^{\prime}, \ldots, j_{M^{\prime}}^{\prime}\right\}, \varepsilon \in \mathbb{Z}_{2}^{N}$ и $k=\varepsilon_{i}(\bmod 2), k^{\prime}=\varepsilon_{i^{\prime}}(\bmod 2)$.

Перейдем к изучению свойств блоховских функций из ядра $L_{\varepsilon}^{\gamma}\left[\begin{array}{l}i \\ k\end{array}\right]_{\mu}$. Далее будем обозначать символом $\psi_{\varepsilon}^{\gamma}\left[\begin{array}{l}i \\ k\end{array}\right](\mu, m)$ для общего $\mu \in X_{\varepsilon}^{\gamma}\left[\begin{array}{l}i \\ k\end{array}\right]$ вектор из $W_{\varepsilon}^{M}(\mu)$, такой, что $\operatorname{ker} L_{\varepsilon}^{\gamma}\left[\begin{array}{l}i \\ k\end{array}\right]_{\mu}=\left\langle\psi_{\varepsilon}^{\gamma}\left[\begin{array}{l}i \\ k\end{array}\right](\mu, \cdot)\right\rangle$, и в выражении $\psi_{\varepsilon}^{\gamma}\left[\begin{array}{l}i \\ k\end{array}\right](m)$ всегда $m \in \Gamma_{1-\varepsilon}^{M}$. 
Отметим, что $\psi_{\varepsilon}^{\gamma}\left[\begin{array}{l}i \\ \gamma\end{array}\right] \in \operatorname{ker} L_{\varepsilon}^{\gamma}\left[\begin{array}{l}i \\ k\end{array}\right]_{\mu}$ определен с точностью до умножения на константу, но отношение $\psi_{\varepsilon}^{\gamma}\left[\begin{array}{l}i \\ k\end{array}\right](m) / \psi_{\varepsilon}^{\gamma}\left[\begin{array}{l}i \\ k\end{array}\right]\left(m^{\prime}\right)$ является корректно определенной мероморфной функцией на $X_{\varepsilon}^{\gamma}\left[\begin{array}{l}i \\ k\end{array}\right]$. Исследуем поведение этой функции в окрестности $X_{\varepsilon}^{\gamma}\left[\begin{array}{l}i, r \\ k, p\end{array}\right]$. Для этого нам понадобится следующая формула:

$$
\frac{\partial R_{\varepsilon}^{\gamma}\left[\begin{array}{l}
i \\
k
\end{array}\right]}{\partial a(\zeta ; n)} / \frac{\partial R_{\varepsilon}^{\gamma}\left[\begin{array}{l}
i \\
k
\end{array}\right]}{\partial a(\xi ; n)}=\frac{\psi_{\varepsilon}^{\gamma}\left[\begin{array}{l}
i \\
k
\end{array}\right]\left(n_{j}+\zeta_{j}\right)}{\psi_{\varepsilon}^{\gamma}\left[\begin{array}{l}
i \\
k
\end{array}\right]\left(n_{j}+\xi_{j}\right)},
$$

где $n_{i}=k, \zeta_{i}=\xi_{i}=\gamma, n_{j} \in \Gamma_{\varepsilon}^{M}$, которая является непосредственным следствием структуры матрицы $\mathbf{M}_{\varepsilon}^{\gamma}\left[\begin{array}{l}i \\ k\end{array}\right]$. Обозначим символом $W_{R}^{\gamma}\left[\begin{array}{l}i \\ k\end{array}\right]$ множество операторов $L$, таких, что

$$
\left.\frac{\partial R_{\varepsilon}^{\gamma, \gamma^{\prime}}\left[\begin{array}{c}
i, r \\
k, p
\end{array}\right]}{\partial a(\xi ; n)}\right|_{X_{\varepsilon}^{\gamma, \gamma^{\prime}}\left[\begin{array}{l}
i, r \\
k, p
\end{array}\right]} \not \equiv 0
$$

при любых $\varepsilon \in \mathbb{Z}_{2}^{N}, r, p, \gamma^{\prime} \in \mathbb{Z}$ и $\xi_{i}=\gamma, \xi_{r}=\gamma^{\prime}, n_{i}=k, n_{j} \in \Gamma_{\varepsilon}^{M}$, символом $U_{R}^{\gamma}\left[\begin{array}{l}i \\ k\end{array}\right]-$ множество операторов $L$, таких, что

$$
\left.\frac{\partial R_{\varepsilon}^{\gamma}\left[\begin{array}{l}
i \\
k
\end{array}\right](j)}{\partial a(\xi ; n)} \mu_{q}^{\bar{\gamma}+\delta_{1-2 \varepsilon_{r}}^{\bar{\gamma}} T_{i, r}^{\prime}}\right|_{X_{\varepsilon}^{\gamma, \bar{\gamma}\left[\begin{array}{l}
i, z \\
k, p
\end{array}\right]}} \not \equiv 0,
$$

где $j_{q}=r, T_{i, r}^{\prime}=\left(T_{r}^{\prime}\right)^{-1} \prod_{a=1}^{M} T_{j_{a}}^{\prime}, \xi_{r}=-\bar{\gamma}, \xi_{i}=\gamma, n_{i}=k, n_{j} \in \Gamma_{\varepsilon}^{M}$ при любых $\varepsilon \in \mathbb{Z}_{2}^{N}, z, r, p, \bar{\gamma} \in \mathbb{Z}$, таких, что либо $z=r$, либо $p \neq m_{r}$, а символом $U_{t r}^{\gamma}\left[\begin{array}{l}i \\ k\end{array}\right]-$ множество операторов $L$, таких, что при любых $\varepsilon \in \mathbb{Z}_{2}^{N}, r \in \mathbb{Z}$ выполнено следующее условие: $X_{\varepsilon}^{\gamma}\left[\begin{array}{l}i, r \\ k, p\end{array}\right]=X_{\varepsilon}^{\gamma}\left[\begin{array}{l}i, r \\ k, p^{\prime}\end{array}\right]$ тогда и только тогда, когда $p=p^{\prime}\left(\bmod 2 T_{r}^{\prime}\right)$. Введенные множества $U_{R}^{\gamma}\left[\begin{array}{l}i \\ k\end{array}\right], W_{R}^{\gamma}\left[\begin{array}{l}i \\ k\end{array}\right], U_{t r}^{\gamma}\left[\begin{array}{l}i \\ k\end{array}\right]$ являются открытыми по Зарисскому, и их непустота будет доказана в лемме 4 .

ПРЕДЛОЖЕНИЕ 1. Для $L \in U_{s m}^{\gamma}\left[\begin{array}{l}i \\ k\end{array}\right] \cap U_{R}^{\gamma}\left[\begin{array}{l}i \\ k\end{array}\right] \cap W_{R}^{\gamma}\left[\begin{array}{l}i \\ k\end{array}\right] \cap U_{t r}^{\gamma}\left[\begin{array}{l}i \\ k\end{array}\right]$ выполняются следующие соотношения:

$$
\begin{aligned}
& \left(\frac{\psi_{\varepsilon}^{\gamma}\left[\begin{array}{l}
i \\
k
\end{array}\right]\left(m^{1}\right)}{\psi_{\varepsilon}^{\gamma}\left[\begin{array}{l}
i \\
k
\end{array}\right]\left(m^{0}\right)}\right)=\mathscr{D}_{m^{1}}^{\gamma}\left[\begin{array}{c}
i \\
k
\end{array}\right]-\mathscr{D}_{m^{0}}^{\gamma}\left[\begin{array}{c}
i \\
k
\end{array}\right]-Q^{\gamma}\left[\begin{array}{l}
i \\
k
\end{array}\right]\left[\begin{array}{c}
m^{1} \\
m^{0}
\end{array}\right]+Q^{\gamma}\left[\begin{array}{c}
i \\
k
\end{array}\right]\left[\begin{array}{c}
m^{0} \\
m^{1}
\end{array}\right], \\
& Q^{\gamma}\left[\begin{array}{c}
i \\
k
\end{array}\right]\left[\begin{array}{c}
m^{1} \\
m^{0}
\end{array}\right]=\sum_{r=1}^{M} \sum_{p=\left(m_{r}^{0}-\varepsilon_{r}^{\prime}\right) / 2}^{\left(m_{r}^{1}-\varepsilon_{r}^{\prime}\right) / 2-1}\left(X_{\tilde{\varepsilon}}^{\gamma, 1}\left[\begin{array}{c}
i, j_{r} \\
k, 2 p+\varepsilon_{r}
\end{array}\right]-X_{\tilde{\varepsilon}}^{\gamma,-1}\left[\begin{array}{c}
i, j_{r} \\
k, 2 p+\varepsilon_{r}
\end{array}\right]\right),
\end{aligned}
$$

где $m, m^{0}, m^{1} \in \Gamma_{\varepsilon^{\prime}}^{M}$,

$$
\mathscr{D}_{m}^{\gamma}\left[\begin{array}{l}
i \\
k
\end{array}\right]=\sum_{\mathscr{C}} \mathscr{C} \max _{0 \leqslant p<T_{j}^{\prime}} \operatorname{ord}_{\mathscr{C}}\left(\frac{\psi_{\varepsilon}^{\gamma}\left[\begin{array}{l}
i \\
k
\end{array}\right](m)}{\psi_{\varepsilon}^{\gamma}\left[\begin{array}{l}
i \\
k
\end{array}\right]\left(\varepsilon^{\prime}+2 p\right)}\right),
$$

причем суммирование идет по всем конечным дивизорам $\mathscr{C} \subset X_{\varepsilon}^{\gamma}\left[\begin{array}{l}i \\ k\end{array}\right], \tilde{\varepsilon}=$ $\left(\varepsilon_{1}, \ldots, \varepsilon_{r-1}, \varepsilon_{r+1}, \ldots, \varepsilon_{M}\right) u \varepsilon^{\prime}=1-\varepsilon$.

Напомним, что дивизор $\mathscr{C}$ конечен тогда и только тогда когда он не имеет неприводимых компонент на бесконечности, т. е. $\operatorname{dim} X_{\infty, \varepsilon}^{\gamma}\left[\begin{array}{l}i \\ k\end{array}\right] \cap \mathscr{C}<M-1$. 
ДокАЗАТЕЛЬство. Теперь нетрудно видеть, что утверждение предложения эквивалентно выполнению следующей формулы:

$$
\operatorname{ord}_{X_{\tilde{\varepsilon}}^{\gamma^{\prime}}\left[\begin{array}{l}
i, r \\
k, p
\end{array}\right]} \frac{\psi_{\varepsilon}^{\gamma}\left[\begin{array}{l}
i \\
k
\end{array}\right]\left(m+2 \bar{\gamma} e_{d}\right)}{\psi_{\varepsilon}^{\gamma}\left[\begin{array}{l}
i \\
k
\end{array}\right](m)}=-\delta_{j_{d}}^{r} \delta_{m_{r}+\bar{\gamma}}^{p},
$$

где $\tilde{\varepsilon}=\left(\varepsilon_{1}, \ldots, \varepsilon_{s-1}, \varepsilon_{s+1}, \ldots, \varepsilon_{M}\right), j_{s}=r$ при произвольных $\bar{\gamma}, r, p, d \in \mathbb{Z}$ и $\gamma^{\prime}=(\gamma, \bar{\gamma})$.

Пусть $\zeta=\xi+2 \bar{\gamma} e_{d}, \xi_{d}=-\bar{\gamma}, n_{i}=k, \xi_{i}=\gamma, n_{j}=m-\xi$.

Рассмотрим сначала случай $j_{d} \neq t$. Перепишем формулу (10) с помощью (7) с учетом того, что $L \in U_{t r}^{\gamma}\left[\begin{array}{l}i \\ k\end{array}\right]$ :

$$
\begin{aligned}
\left.\frac{\psi_{\varepsilon}^{\gamma}\left[\begin{array}{c}
i \\
k
\end{array}\right]\left(m+2 \bar{\gamma} e_{d}\right)}{\psi_{\varepsilon}^{\gamma}\left[\begin{array}{l}
i \\
k
\end{array}\right](m)}\right|_{X_{\tilde{\varepsilon}}^{\gamma^{\prime}}\left[\begin{array}{l}
i, r \\
k, p
\end{array}\right]} & =\frac{\partial R_{\varepsilon}^{\gamma}\left[\begin{array}{l}
i \\
k
\end{array}\right]}{\partial a(\zeta ; n)} /\left.\frac{\partial R_{\varepsilon}^{\gamma}\left[\begin{array}{l}
i \\
k
\end{array}\right]}{\partial a(\xi ; n)}\right|_{X_{\tilde{\varepsilon}}^{\gamma^{\prime}}\left[\begin{array}{l}
i, r \\
k, p
\end{array}\right]} \\
& =\frac{\partial R_{\tilde{\varepsilon}}^{\gamma^{\prime}\left[\begin{array}{c}
i, r \\
k, p
\end{array}\right]}}{\partial a(\zeta ; n)} /\left.\frac{\partial R_{\tilde{\varepsilon}}^{\gamma^{\prime}}\left[\begin{array}{c}
i, r \\
k, p
\end{array}\right]}{\partial a(\xi ; n)}\right|_{X_{\tilde{\varepsilon}}^{\gamma^{\prime}}\left[\begin{array}{l}
i, r \\
k, p
\end{array}\right]}
\end{aligned}
$$

при $\xi_{r}=\zeta_{r}=\bar{\gamma}$. Теперь утверждение очевидным образом следует из определения $W_{R}^{\gamma}\left[\begin{array}{l}i \\ k\end{array}\right]$.

Рассмотрим далее случай $r=j_{d}$. Докажем утверждение в случае $\varepsilon_{r}=1$, $\bar{\gamma}=-1, j_{1}=r$ (в остальных случаях доказательство проводится аналогично). Так как $L \in U_{t r}^{\gamma}\left[\begin{array}{l}i \\ k\end{array}\right]$, то существует $q \neq 1$, такое, что $\partial R_{\varepsilon}^{\gamma}\left[\begin{array}{l}i \\ k\end{array}\right] / \partial \mu_{q} \neq \equiv 0$; следовательно, $\mu_{1}, \ldots, \mu_{q-1}, \mu_{q+1}, \ldots, \mu_{M}$ являются координатами на $X_{\varepsilon}^{\gamma}\left[\begin{array}{l}i \\ k\end{array}\right]$ в окрестности $X_{\tilde{\varepsilon}}^{\gamma^{\prime}}\left[\begin{array}{l}i, r \\ k, p\end{array}\right]$ и в этих координатах $X_{\tilde{\varepsilon}}^{\gamma^{\prime}}\left[\begin{array}{l}i, r \\ k, p\end{array}\right]$ задается уравнением $\mu_{1}=0$. Используя формулы (10) и (7), выпишем разложение в терминах этих координат:

$$
\begin{aligned}
& \frac{\psi_{\varepsilon}^{\gamma}\left[\begin{array}{l}
i \\
k
\end{array}\right]\left(m+2 \bar{\gamma} e_{d}\right)}{\psi_{\varepsilon}^{\gamma}\left[\begin{array}{l}
i \\
k
\end{array}\right](m)}=\frac{\partial R_{\varepsilon}^{\gamma}\left[\begin{array}{l}
i \\
k
\end{array}\right]}{\partial a(\zeta ; n)} / \frac{\partial R_{\varepsilon}^{\gamma}\left[\begin{array}{l}
i \\
k
\end{array}\right]}{\partial a(\xi ; n)} \\
& =\left(\frac{\partial R_{\varepsilon}^{\gamma}\left[\begin{array}{l}
i \\
k
\end{array}\right]}{\partial a(\xi ; n)} \mu_{1}^{-1}\right)^{-1} \mu_{1}^{-1}\left(\frac{\partial R_{\tilde{\varepsilon}}^{\gamma^{\prime}}\left[\begin{array}{c}
i, r \\
k, n_{r}-1
\end{array}\right]}{\partial a(\zeta ; n)}\left(R_{\tilde{\varepsilon}}^{\gamma^{\prime}}\left[\begin{array}{c}
i, r \\
k, n_{r}-1
\end{array}\right]\right)^{-1} \prod_{s=0}^{T_{r}^{\prime}-1} R_{\tilde{\varepsilon}}^{\gamma^{\prime}}\left[\begin{array}{c}
i, r \\
k, s
\end{array}\right]+\mu_{1} Q(\mu)\right),
\end{aligned}
$$

где

$$
\operatorname{ord}_{X_{\tilde{\varepsilon}}^{\gamma^{\prime}\left[\begin{array}{l}
i, r \\
k, p
\end{array}\right]}} Q(\mu)=\operatorname{ord}_{X_{\tilde{\varepsilon}}^{\gamma}\left[\begin{array}{l}
i, r \\
k, p
\end{array}\right]} \frac{\partial R_{\tilde{\varepsilon}}^{\gamma}\left[\begin{array}{l}
i \\
k
\end{array}\right]}{\partial a(\zeta ; n)} \mu_{1}^{-1}=0 .
$$

Элементарным анализом полученной формулы в окрестности $\mu_{1}=0$ получаем утверждение предложения.

Заметим, что из соотношения (13) следует равенство

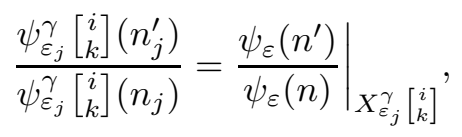

а из (12) и (9), (11) следуют равенства

$$
\begin{aligned}
Q^{\gamma}\left[\begin{array}{l}
i \\
k
\end{array}\right]\left[\begin{array}{l}
n_{j}^{\prime} \\
n_{j}
\end{array}\right] & =Q\left[\begin{array}{l}
n^{\prime} \\
n
\end{array}\right] X_{\varepsilon_{j}}^{\gamma}\left[\begin{array}{l}
i \\
k
\end{array}\right], \\
\mathscr{D}_{n_{j}}^{\gamma}\left[\begin{array}{l}
i \\
k
\end{array}\right] & =\mathscr{D}_{n} X_{\varepsilon_{j}}^{\gamma}\left[\begin{array}{l}
i \\
k
\end{array}\right],
\end{aligned}
$$


где $n_{i}=k+\gamma, n, n^{\prime} \in \Gamma_{1-\varepsilon}^{N}$. Наличие индуктивной структуры спектральных данных фактически заключается в этих трех формулах, которые связывают спектральные данные и собственную функцию оператора $L$ со спектральными данными и собственной функцией оператора $L^{\gamma}\left[\begin{array}{l}i \\ k\end{array}\right]$.

Введем еще одно обозначение: $\Delta_{\varepsilon}^{\gamma}\left[\begin{array}{l}i \\ k\end{array}\right]\left(m, m^{\prime}\right)$ для алгебраического дополнения к $\mathbf{M}_{\varepsilon}^{\gamma}\left[\begin{array}{l}i \\ k\end{array}\right]_{u, w}$, где $u=1+\sum_{b=1}^{M} m_{b} \prod_{t=b+1}^{M} T_{j_{t}}^{\prime}$ и $w=1+\sum_{b=1}^{M} m_{b}^{\prime} \prod_{t=b+1}^{M} T_{j_{t}}^{\prime}$, $0 \leqslant m, m^{\prime}<T_{j}^{\prime}$. Определим следующий дивизор на $X_{\varepsilon}^{\gamma}\left[\begin{array}{l}i \\ k\end{array}\right]$ :

$$
\mathscr{E}_{\varepsilon}^{\gamma}\left[\begin{array}{l}
i \\
k
\end{array}\right]=\sum_{\mathscr{C}} \mathscr{C}_{0 \leqslant m, m^{\prime}<T_{j}^{\prime}}\left(\operatorname{ord}_{\mathscr{C}} \Delta_{\varepsilon}^{\gamma}\left[\begin{array}{l}
i \\
k
\end{array}\right]\left(m, m^{\prime}\right)\right),
$$

где суммирование идет по всем конечным дивизорам $\mathscr{C} \subset X_{\varepsilon}^{\gamma}\left[\begin{array}{l}i \\ k\end{array}\right]$.

Теперь введем следующий важный дифференциал на $X_{\varepsilon}^{\gamma}\left[\begin{array}{l}i \\ k\end{array}\right]$ :

$$
\Omega_{\varepsilon ; n ; \zeta}^{\gamma}\left[\begin{array}{l}
i \\
k
\end{array}\right]=\left(\frac{\partial R_{\varepsilon}^{\gamma}\left[\begin{array}{l}
i \\
k
\end{array}\right]}{\partial \hat{\mu}_{r}}\right)^{-1} \frac{\partial R_{\varepsilon}^{\gamma}\left[\begin{array}{l}
i \\
k
\end{array}\right]}{\partial a(\zeta ; n)} \frac{\bigwedge_{p \neq r} d \hat{\mu}_{p}}{\prod_{p=1}^{M} \hat{\mu}_{p}}
$$

$\gamma=\zeta_{i}, n_{i}=k, n_{j} \in \Gamma_{\varepsilon}^{M}, \hat{\mu}_{r}=\mu_{r}^{1-2 \varepsilon_{j_{r}}}$.

ПреДЛОЖенИЕ 2. Для любого $L \in U_{R}^{\gamma}\left[\begin{array}{l}i \\ k\end{array}\right] \cap W_{R}^{\gamma}\left[\begin{array}{l}i \\ k\end{array}\right] \cap U_{i r}^{\gamma}\left[\begin{array}{l}i \\ k\end{array}\right] \cap U_{s m}^{\gamma}\left[\begin{array}{l}i \\ k\end{array}\right] \cap U_{t r}^{\gamma}\left[\begin{array}{l}i \\ k\end{array}\right]$ выполняется равенство

$$
\left(\Omega_{\varepsilon ; n ; \zeta}^{\gamma}\left[\begin{array}{l}
i \\
k
\end{array}\right]\right)=\mathscr{E}_{\varepsilon}^{\gamma}\left[\begin{array}{l}
i \\
k
\end{array}\right]+\mathscr{D}_{n_{j}+\zeta_{j}}^{\gamma}\left[\begin{array}{l}
i \\
k
\end{array}\right]+\sigma\left(\mathscr{D}_{n_{j}}^{-\gamma}\left[\begin{array}{c}
i \\
k+\gamma
\end{array}\right]\right)-\sum_{r=1}^{M} X_{\tilde{\varepsilon}}^{\gamma, \zeta_{j_{r}}}\left[\begin{array}{c}
i, j_{r} \\
k, n_{j_{f}}
\end{array}\right],
$$

где $\tilde{\varepsilon}=\left(\varepsilon_{1}, \ldots, \varepsilon_{r-1}, \varepsilon_{r+1}, \ldots, \varepsilon_{M}\right)$.

ДокАЗАТЕЛЬство. Элементарным манипулированием с разложениями в ряды Лорана в окрестности $X_{\varepsilon}^{\infty, \gamma}\left[\begin{array}{l}i \\ k\end{array}\right]$ (аналогичным проведенному в предложении 1) получаем

$$
\left(\Omega_{\varepsilon ; n ; \zeta}^{\gamma}\left[\begin{array}{l}
i \\
k
\end{array}\right]\right) \sim-\sum_{r=1}^{M} X_{\tilde{\varepsilon}}^{\gamma, \zeta_{j_{r}}}\left[\begin{array}{c}
i, j_{r} \\
k, n_{j_{r}}
\end{array}\right]+\widetilde{\mathscr{D}},
$$

где $\widetilde{\mathscr{D}}>0-$ эффективный конечный дивизор.

Вычислим $\operatorname{ord}_{\mathscr{C}}\left(\widetilde{D}-\mathscr{D}_{n_{j}+\zeta_{j}}^{\gamma}\left[\begin{array}{l}i \\ k\end{array}\right]-\sigma\left(\mathscr{D}_{n_{j}}^{-\gamma}\left[\begin{array}{c}i \\ k+\gamma\end{array}\right]\right)\right)$, где $\mathscr{C} \subset X_{\varepsilon}^{\gamma}\left[\begin{array}{l}i \\ k\end{array}\right]-$ конечный дивизор. Проведем рассуждения для $\varepsilon=0, \zeta=1$ (в оставшихся случаях рассуждения аналогичны). В силу $T_{j}$-периодичности можно без ограничения общности считать, что $0 \leqslant n_{j}<2 T_{j}^{\prime}$. Из структуры матрицы $\mathbf{M}_{\varepsilon}^{\gamma}\left[\begin{array}{l}i \\ k\end{array}\right]$ следует равенство $\partial R_{\varepsilon}^{\gamma}\left[\begin{array}{l}i \\ k\end{array}\right] / \partial a(\zeta ; n)=\mu^{\kappa} \Delta\left(n_{j} / 2, n_{j} / 2\right)$, где $\kappa \in \mathbb{Z}^{M}$. Таким образом, в силу голоморфности дифференциала $\left(\partial R_{\varepsilon}^{\gamma}\left[\begin{array}{l}i \\ k\end{array}\right] / \partial \hat{\mu}\right)^{-1} \prod_{p \neq r} d \hat{\mu}_{p}$ на $X_{\varepsilon}^{\text {aff, }, \gamma}\left[\begin{array}{l}i \\ k\end{array}\right]$ и формулы $(5)$ имеем

$$
\begin{aligned}
\operatorname{ord}_{\mathscr{C}} \widetilde{D} & =\min _{m^{\prime}, m^{\prime \prime}} \operatorname{ord}_{\mathscr{C}}\left(\frac{\psi^{\gamma}\left[\begin{array}{l}
i \\
k
\end{array}\right]\left(2 m^{\prime}+1\right)}{\psi^{\gamma}\left[\begin{array}{l}
i \\
k
\end{array}\right](2 m+1)} \frac{\psi^{\gamma}\left[\begin{array}{l}
i \\
k
\end{array}\right]\left(2 m^{\prime \prime}+1\right)}{\psi^{\gamma}\left[\begin{array}{l}
i \\
k
\end{array}\right](2 m+1)} \Delta_{\varepsilon}^{\gamma}\left[\begin{array}{c}
i \\
k
\end{array}\right](m, m)\right) \\
& =\min _{m^{\prime \prime}, m^{\prime}} \operatorname{ord}_{\mathscr{C}}\left(\frac{\Delta_{\varepsilon}^{\gamma}\left[\begin{array}{l}
i \\
k
\end{array}\right]\left(m^{\prime \prime}, m^{\prime}\right)}{\Delta_{\varepsilon}^{\gamma}\left[\begin{array}{l}
i \\
k
\end{array}\right]\left(m^{\prime \prime}, m\right)} \frac{\Delta_{\varepsilon}^{\gamma}\left[\begin{array}{l}
i \\
k
\end{array}\right]\left(m^{\prime \prime}, m\right)}{\Delta_{\varepsilon}^{\gamma}\left[\begin{array}{l}
i \\
k
\end{array}\right](m, m)} \Delta_{\varepsilon}^{\gamma}\left[\begin{array}{c}
i \\
k
\end{array}\right](m, m)\right)=\operatorname{ord}_{\mathscr{C}} \mathscr{E}_{\varepsilon}^{\gamma}\left[\begin{array}{l}
i \\
k
\end{array}\right],
\end{aligned}
$$

где $2 m=n_{j}$. 
Символом $U_{E}^{\gamma}\left[\begin{array}{l}i \\ k\end{array}\right]$ мы будем обозначать открытое по Зарисскому множество операторов $L \in U_{s m}^{\gamma}\left[\begin{array}{l}i \\ k\end{array}\right] \cap U_{i r}^{\gamma}\left[\begin{array}{l}i \\ k\end{array}\right] \cap W_{R}^{\gamma}\left[\begin{array}{l}i \\ k\end{array}\right]$, для которых выполнено условие $\mathscr{E}_{\varepsilon}^{\gamma}\left[\begin{array}{l}i \\ k\end{array}\right]=0$ для всех $\varepsilon$.

ЗАмЕчАНиЕ. Для введенных дифференциалов имеет место формула, аналогичная формуле (14):

$$
P . R ._{X_{\tilde{\varepsilon}}^{\gamma, \zeta_{j_{r}}}\left[\begin{array}{c}
i, j_{r} \\
k, n_{j_{r}}
\end{array}\right]} \Omega_{\varepsilon ; n ; \zeta}^{\gamma}\left[\begin{array}{l}
i \\
k
\end{array}\right]=\Omega_{\tilde{\varepsilon} ; n ; \zeta}^{\gamma, \zeta_{j_{r}}}\left[\begin{array}{c}
i, j_{r} \\
k, n_{j_{r}}
\end{array}\right],
$$

где P.R. - обозначение для вычета Пуанкаре (см. [19]). Эта формула отражает наличие индуктивной структуры изучаемой спектральной задачи.

Введем определение данных, совместимых с набором периодов $T$. Пусть $\left\{X_{\varepsilon} \subset\right.$ $\left.\left(\mathbb{C P}^{1}\right)_{\mu}^{N}\right\}$ - набор многообразий, совместимых с набором периодов $T$, и на этом наборе многообразий имеется набор дивизоров $\mathscr{D}_{n}, n \in \mathbb{Z}^{N}$, удовлетворяющих условиям

$$
\begin{gathered}
\mathscr{D}_{n}=\mathscr{D}_{n+T_{r} e_{r}} \\
\mathscr{K}_{X_{\varepsilon}} \sim \mathscr{D}_{n+\zeta}+\sigma\left(\mathscr{D}_{n}\right)-\sum_{r=1}^{N} X_{\tilde{\varepsilon}}^{\zeta_{r}}\left[\begin{array}{c}
r \\
n_{r}
\end{array}\right], \\
\mathscr{D}_{n^{1}}-\mathscr{D}_{n^{0}}-Q\left[\begin{array}{c}
n^{1} \\
n^{0}
\end{array}\right]+Q\left[\begin{array}{c}
n^{0} \\
n^{1}
\end{array}\right] \sim 0
\end{gathered}
$$

где $\tilde{\varepsilon}=\left(\varepsilon_{1}, \ldots, \varepsilon_{r-1}, \varepsilon_{r+1}, \ldots, \varepsilon_{N}\right)$, при всех $n^{0}, n^{1} \in \Gamma_{\varepsilon}^{N}, n, \zeta, \varepsilon \in \mathbb{Z}^{N}, r=$ $1, \ldots, N$ (здесь использована формула $(12)$ при $j=(1, \ldots, N))$. Такой набор $\left\{X_{\varepsilon} \subset\left(\mathbb{C P}^{1}\right)_{\mu}^{N}, \mathscr{D}_{n}\right\}$ мы будем называть набором данных, совместимых $c$ набором периодов $T$. Множество наборов данных, совместимых с набором периодов $T$, будем обозначать через $\mathfrak{M}_{T}$.

Если рассматривать систему соотношений (17)-(19) как соотношения между дивизорами, то очевидно, что эта система избыточна, из нее может быть исключено соотношение $(19)$ и все дивизоры $\mathscr{D}_{n}$ будут выражаться через дивизоры $\mathscr{D}_{\varepsilon}$, $\varepsilon_{h}=0$, которые, в свою очередь, удовлетворяют соотношениям

$$
\begin{aligned}
& \mathscr{K}_{X_{\varepsilon}} \sim \mathscr{D}_{\varepsilon^{\prime}}+\sigma\left(\mathscr{D}_{\varepsilon}\right)-\sum_{r=1}^{N} X_{\tilde{\varepsilon}}^{1}\left[\begin{array}{c}
r \\
\varepsilon_{r}
\end{array}\right]+\sum_{r=1}^{N-S} \sum_{p=0}^{\left(T_{h_{r}}-1\right) / 2}\left(X_{\tilde{\varepsilon}^{\prime}}^{1}\left[\begin{array}{c}
h_{r} \\
2 p+1
\end{array}\right]-X_{\tilde{\varepsilon}^{\prime}}^{-1}\left[\begin{array}{c}
h_{r} \\
2 p+1
\end{array}\right]\right), \\
& \mathscr{D}_{\varepsilon}-Q\left[\begin{array}{c}
\varepsilon \\
\varepsilon+2 n
\end{array}\right]+Q\left[\begin{array}{c}
\varepsilon+2 n \\
\varepsilon
\end{array}\right]>0, \quad 0 \leqslant n<T^{\prime},
\end{aligned}
$$

где $\varepsilon_{l}+\varepsilon_{l}^{\prime}=1, \varepsilon_{h}=\varepsilon_{h}^{\prime}=0, \tilde{\varepsilon}^{\prime}=1-\tilde{\varepsilon}$ и предполагается, что $j=(1, \ldots, N)$. Следовательно, если мы хотим рассматривать $\mathfrak{M}_{T}$ как пространство спектральных данных прямой спектральной задачи, то элементы $\mathfrak{m} \in \mathfrak{M}_{T}$ следует интерпретировать как наборы $\left\{X_{\varepsilon} \subset\left(\mathbb{C P}^{1}\right)_{\mu}^{N}, \mathscr{D}_{\varepsilon}\right\}, \varepsilon_{h}=0$, где $\left\{X_{\varepsilon} \subset\left(\mathbb{C P}^{1}\right)_{\mu}^{N}\right\}$ совместим с набором периодов $T$, а для $\mathscr{D}_{\varepsilon}$ верны соотношения $(20),(21)$. Отметим, что пространство спектральных данных для многомерной задачи имеет описание, сходное с описанием спектральных данных для двумерных задач $[6,8]$; единственным отличием является условие (21), которое в двумерном случае выполнено автоматически в силу теоремы Римана-Роха, но в многомерном случае это условие является сильным ограничением на $\mathscr{D}_{\varepsilon}$ и на $X_{\varepsilon}$.

Следующее предложение формализует утверждение о наличии индуктивной структуры на спектральных данных. 
ПРЕДЛОЖЕНИЕ 3. Если $\left\{X_{\varepsilon} \subset\left(\mathbb{C P}^{1}\right)_{\mu}^{N}, \mathscr{D}_{\varepsilon}\right\} \in \mathfrak{M}_{T}, \operatorname{mo}\left\{X_{\varepsilon}^{\gamma}\left[\begin{array}{l}i \\ k\end{array}\right] \subset\left(\mathbb{C P}^{1}\right)_{\mu_{j}}^{M}, \mathscr{D}_{\varepsilon_{j}}^{\gamma}\left[\begin{array}{l}i \\ k\end{array}\right]\right\}$ $\in \mathfrak{M}_{T_{j}}$ (m.е. $\left\{X_{\varepsilon}^{\gamma}\left[\begin{array}{l}i \\ k\end{array}\right] \subset\left(\mathbb{C P}^{1}\right)_{\mu_{j}}^{M}, \mathscr{D}_{\varepsilon_{j}}^{\gamma}\left[\begin{array}{l}i \\ k\end{array}\right]\right\}$ являются спектральными данными $T_{j}$-периодической спектральной задачи), где $\mathscr{D}_{n_{j}}^{\gamma}\left[\begin{array}{l}i \\ k\end{array}\right]$ определено формулой (16).

ДокАЗАТЕЛЬСтво. Достаточно показать, что $\left\{X_{\varepsilon}^{\bar{\gamma}}\left[\begin{array}{l}r \\ p\end{array}\right] \subset\left(\mathbb{C P}^{1}\right)_{\bar{\mu}}^{N-1}, \mathscr{D}_{\varepsilon, m}^{\bar{\gamma}}\left[\begin{array}{l}r \\ p\end{array}\right]\right\} \in$ $\mathfrak{M}_{\bar{T}}, \bar{T}=\left(T_{1}, \ldots, T_{r-1}, T_{r+1}, \ldots, T_{N}\right)$. Для доказательства этого факта заметим, что по формуле присоединения (см. [19]) $\mathscr{K}_{X_{\varepsilon}^{\bar{\gamma}}\left[\begin{array}{l}r \\ p\end{array}\right]} \sim\left(\mathscr{K}_{X_{\varepsilon}}+X_{\varepsilon}^{\bar{\gamma}}\left[\begin{array}{l}r \\ p\end{array}\right]\right) X_{\varepsilon}^{\bar{\gamma}}\left[\begin{array}{l}r \\ p\end{array}\right]$; при $n_{r}=p+\bar{\gamma}, \zeta_{r}=-\bar{\gamma}$, подставляя (18) для $T$-периодической задачи в эту формулу и используя (16), (9), получаем выполнение условия (18) для $T^{\prime}$-периодической задачи. Выполнение условия (19) тривиально следует из (15), (9).

На пространстве операторов $L$ действует калибровочная группа. Для любой $T$-периодической функции $g \in V(1), g(n) \neq 0$ для всех $n \in \mathbb{Z}^{N}$, определено отображение $F_{g}: F_{g}(L)=L^{\prime}, a^{\prime}(n, \zeta)=g(n) g(n+\zeta) a(n, \zeta)$. Фактор пространства операторов $L$ по действию калибровочной группы будем обозначать через $\mathfrak{L}_{T}$; образы открытых множеств $U_{s m}, U_{i r}, W_{R}, U_{R}, U_{E}, U_{t r}$ при такой факторизации будем обозначать теми же символами. Пусть $\mathfrak{U}=\bigcap_{i, k, \gamma}\left(U_{s m}^{\gamma}\left[\begin{array}{l}i \\ k\end{array}\right] \cap U_{i r}^{\gamma}\left[\begin{array}{l}i \\ k\end{array}\right] \cap\right.$ $\left.W_{R}^{\gamma}\left[\begin{array}{l}i \\ k\end{array}\right] \cap U_{R}^{\gamma}\left[\begin{array}{l}i \\ k\end{array}\right] \cap U_{E}^{\gamma}\left[\begin{array}{l}i \\ k\end{array}\right] \cap U_{t r}^{\gamma}\left[\begin{array}{l}i \\ k\end{array}\right]\right)$; тогда, как следует из доказанной последовательности лемм, на множестве $\mathfrak{U}$ определено отображение $\mathfrak{D}: \mathfrak{L}_{T} \rightarrow \mathfrak{M}_{T}$, являющееся прямой задачей рассеяния. В следующем параграфе будет доказана плотность множества $\mathfrak{U}$ в $\mathfrak{L}_{T}$ и найдено обратное к $\mathfrak{D}$ отображение $\mathfrak{I}$.

\section{§3. Обратная задача}

В двумерном случае метод решения обратной задачи рассеяния (в нашем случае решение обратной задачи рассеяния эквивалентно построению отображения I) был предложен Кричевером в работе [6]. Мы используем результаты работы [6] для доказательства плотности множества $\mathfrak{U}$ в $\mathfrak{L}_{T}$, а также для доказательства обратимости отображения $\mathfrak{D}$.

Лемма 2. Пусть $N=2 u h_{\varepsilon}\left[\begin{array}{c}n \\ n^{\prime}\end{array}\right]=H^{0}\left(X_{\varepsilon}, \mathscr{O}\left(Q\left[\begin{array}{c}n \\ n^{\prime}\end{array}\right]+\mathscr{D}_{n^{\prime}}\right)\right) ;$ mогда $\mathfrak{M}_{T} \neq \varnothing u$ для общего представителя $\mathfrak{m} \in \mathfrak{M}_{T}$

$$
\operatorname{dim} h_{\varepsilon}\left[\begin{array}{c}
n \\
n^{\prime}
\end{array}\right]=1
$$

ДоказАтЕльство. Разберем случай, когда $T_{1}, T_{2}$ четны. В этом случае спектральные данные состоят из кривых $X_{0,0}, X_{0,1}, \sigma\left(X_{0,0}\right), \sigma\left(X_{0,1}\right)$ и дивизоров $\mathscr{D}_{\varepsilon}$. При этом $X_{0,0}, X_{0,1}$ являются кривыми степени $\left(T_{1}^{\prime}, T_{2}^{\prime}\right)$, определяемыми уравнениями с общими коэффициентами, а следовательно, $X_{0,0}, X_{0,1}$ - гладкие кривые рода $\left(T_{1}^{\prime}-1\right)\left(T_{2}^{\prime}-1\right)$. Из $(18)$ следует, что $\left|\mathscr{D}_{n}\right|=\left(T_{1}^{\prime}-1\right)\left(T_{2}^{\prime}-1\right)$. Выберем $m_{e} \in \Gamma_{1,1-e}^{2}, e=0,1$; тогда соотношения $(18),(19)$ позволяют выразить все $\mathscr{D}_{n}$ через $\mathscr{D}_{m_{e}}$. Более того, если мы выберем два общих положительных дивизора $\mathscr{D}_{m_{e}}$ степени $\left(T_{1}^{\prime}-1\right)\left(T_{2}^{\prime}-1\right)$, то в силу равенства $\left|Q_{\varepsilon}\left[\begin{array}{c}n^{1} \\ n^{0}\end{array}\right]\right|=\left|Q_{\varepsilon}\left[\begin{array}{c}n^{1} \\ n^{0}\end{array}\right]\right|=0$ и теоремы Римана-Роха имеем $\operatorname{dim} h_{0, e}\left[\begin{array}{c}n \\ m_{e}\end{array}\right]=1$, и по теореме Якоби (см. [19]) для $\mathscr{D}_{m_{e}}$ существуют и единственны такие положительные дивизоры $\mathscr{D}_{n}$, для которых выполнены условия (18), (19). Так как $\mathscr{D}_{m_{e}}$ выбраны общими, то по теореме Римана-Роха выполняется равенство (22). Оставшиеся случаи разбираются аналогично. 
Tеорема 1. Пусть $N=2 ;$ тогда множество $\mathfrak{U}$ плотно в $\mathfrak{L}_{T}$ и отображение $\mathfrak{D}: \mathfrak{L}_{T} \rightarrow \mathfrak{M}_{T}$ обратимо на некотором всюду плотном множестве. Отображение $\mathfrak{I}=\mathfrak{D}^{-1}$ может быть описано с помощью $\theta$-функций кривых $X_{\varepsilon}$.

ДокАЗАтЕЛЬство. Разберемся сначала со случаем, когда $T_{1}, T_{2}$ четны. Выберем $m_{e} \in \Gamma_{1,1-e}, e=0,1$. Выберем $T$-блоховские функции $\psi(n)$ так, чтобы выполнялось (11). По набору функций $\varphi_{0, e}=\psi_{0, e}(n) / \psi_{0, e}\left(m_{e}\right) \in h_{0, e}\left[\begin{array}{c}n \\ m_{e}\end{array}\right]$ способом, изложенным в [6], строим оператор $\widetilde{L}_{0, e}$, действующий на $V_{1,1-e}, \widetilde{L}_{0, e} \varphi_{0, e}=$ $\sum_{\left(\zeta_{1}, \zeta_{2}\right)} \tilde{a}(n, \zeta) \varphi_{0, e}(n+\zeta)$, такой, что $\widetilde{L}_{0, e} \varphi_{0, e}=0$. Оператор $\widetilde{L}_{0, e}$ определяется функциями $\varphi_{0, e}$ неоднозначно. Действительно, оператор $\widetilde{L}_{0, e}^{\prime}$ с коэффициентами $\tilde{a}^{\prime}(n, \zeta)=\tilde{a}(n, \zeta) C(n)$, где $C(n)-$ произвольные константы, также удовлетворяет уравнению $\widetilde{L}_{0, e}^{\prime} \varphi_{0, e}=0$. Теперь если мы положим $\tilde{a}(n, \zeta)=\tilde{a}(n+\zeta,-\zeta)$ для $n \in \Gamma_{1,1-e}^{2}, e=0,1$, то получим оператор $\widetilde{L}$, действующий на $V$. Фиксируем $n_{e} \in \Gamma_{0, e}^{2}, e=0,1$. В силу предложения 1 и леммы 2 для собственной функции $\varphi_{1,1-e}, e=0,1, \widetilde{L} \varphi_{1,1-e}=0$, выполняется равенство

$$
\frac{\varphi_{1,1-e}(n)}{\varphi_{1,1-e}\left(n_{e}\right)}=\widehat{C}(n) \frac{\psi_{1,1-e}(n)}{\psi_{1,1-e}\left(n_{e}\right)},
$$

где $\widehat{C}(n)-$ некоторые константы. Положив $a(n, \zeta)=\tilde{a}(n, \zeta) \widehat{C}(n), n \in \Gamma_{0, e}^{2}$, получим оператор $L$, такой, что $L \psi=0$. Произвол в выборе функций $\psi(n)$ при построении оператора $L$ со свойством $L \psi=0$ отвечает действию калибровочной группы. Таким образом, мы получили корректно определенное отображение $\mathfrak{I}: \mathfrak{M}_{T} \rightarrow L_{T}$. При этом из предыдущих рассуждений следует, что существует единственный оператор $L$, нормированный условием $a\left(m_{0} ; 1,1\right)=a\left(m_{1} ; 1,1\right)=1$ и удовлетворящий условию $L \psi=0$. Таким образом, $\mathfrak{I D}=\mathfrak{D} \mathfrak{I}=\mathrm{id}$. Так как для общего $\mathfrak{m} \in \mathfrak{M}_{T}$ имеет место включение $\mathfrak{I}(\mathfrak{m}) \in \mathfrak{U}$, то $\mathfrak{U} \neq \varnothing$.

Построение $\mathfrak{I}$ в оставшихся случаях проводится аналогично. Единственное отличие от уже разобранного случая заключается в том, что если $T_{1}$ нечетен, а $T_{2}$ четен, то $\psi$ удовлетворяет соотношению $(11)$ и $\psi_{0, \varepsilon}(n)=\psi_{1, \varepsilon}\left(n+T_{1} e_{1}\right), \varepsilon=0,1$, а если $T_{1}$ и $T_{2}$ нечетны, то $\psi$ удовлетворяет соотношению $(11)$ и $\psi_{0,0}(n)=$ $\psi_{0,1}\left(n+T_{2} e_{2}\right)=\psi_{1,0}\left(n+T_{1} e_{1}\right)=\psi_{1,1}\left(n+T_{1} e_{1}+T_{2} e_{2}\right)$.

Теперь докажем плотность множества $\mathfrak{U}$ в случае произвольной размерности $N$. Мы будем доказывать это индукцией по $N$. Можно считать, что спектральные многообразия всех операторов $L$ лежат в одном $\left(\mathbb{C P}^{1}\right)^{N}$. Тогда, если мы обозначим символом $X_{\varepsilon}(L)$ спектральное многообразие оператора $L$, то верен следующий факт:

ЛЕмма 3. Для произвольного $\varepsilon \in \mathbb{Z}^{N}$ справедливо соотношение $\bigcap_{L} X_{\varepsilon}(L)=\varnothing$.

ДокАЗАТЕльство. Доказательство будем проводить индукцией по $N$.

Фиксируем произвольные числа $1 \leqslant r \leqslant N, k \in \mathbb{Z}, \kappa \in \mathbb{C}^{*}$ и заметим, что если нам даны два оператора $L$ и $L^{\prime}$, такие, что $a(n ; \zeta)=a^{\prime}(n ; \zeta)$ при $n_{r} \neq k$ $\left(\bmod T_{r}\right)$ и $a(n ; \zeta)=\kappa a(n ; \zeta)$ при $n_{r}=k\left(\bmod T_{r}\right)$, то

$$
\left(\mu_{1}, \ldots, \mu_{r}, \ldots, \mu_{N}\right) \in X_{\varepsilon}^{\mathrm{aff}}\left(L^{\prime}\right) \Longleftrightarrow\left(\mu_{1}, \ldots, \kappa \mu_{r}, \ldots, \mu_{N}\right) \in X_{\varepsilon}^{\mathrm{aff}}(L) .
$$

Значит, если $\mu \in \bigcap_{L} X_{\varepsilon}(L)$, то $\tilde{\mu}=\left(\mu_{1}, \ldots, \kappa \mu_{r}, \ldots, \mu_{N}\right) \in \bigcap_{L} X_{\varepsilon}^{\text {aff }}(L)$ для произвольных $r, \kappa$. Таким образом, получаем, что если $\mu \in \bigcap_{L} X_{\varepsilon}(L)$, то $\mu \in \bigcap_{L} X_{\varepsilon}^{\infty}(L)$. 
Далее мы можем применить предположение индукции, так как $X_{\varepsilon}^{\infty}(L)$ является объединением спектральных многообразий операторов $L_{\varepsilon}^{\gamma}\left[\begin{array}{l}r \\ p\end{array}\right]$.

ЛЕмма 4. При любом $N$ множество $\mathfrak{U}$ плотно в $\mathfrak{L}_{T}$.

ДокАЗАТЕЛЬство. Так как рассматриваемое множество является пересечением открытых по Зарисскому множеств, то достаточно доказать непустоту множеств $U_{s m}, U_{i r}, W_{R}, U_{E}, U_{t r}$.

Тот факт, что $U_{t r} \neq \varnothing$, немедленно следует из леммы 3.

Докажем, что $U_{R} \neq \varnothing$. Будем доказывать это индукцией по $N$. Пусть это не так, т. е. существуют $r, \bar{\gamma} \in \mathbb{Z}, \varepsilon \in \mathbb{Z}_{2}^{N}$, $\zeta$, такие, что

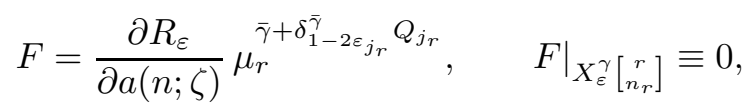

где $Q_{j_{r}}=\left(T_{j_{r}}^{\prime}\right)^{-1} \prod_{a=1}^{N} T_{a}^{\prime}$. Пусть $s \neq r$; тогда

$$
\left.F\right|_{X_{\tilde{\varepsilon}}^{\gamma^{\prime}}\left[\begin{array}{c}
s \\
n_{s}
\end{array}\right]}=\frac{\partial R_{\tilde{\varepsilon}}^{\gamma^{\prime}\left[\begin{array}{c}
s \\
n_{s}
\end{array}\right]}}{\partial a(n ; \zeta)} \mu_{r}^{\bar{\gamma}+v}=\frac{\partial R_{\tilde{\varepsilon}}^{\gamma^{\prime}}\left[\begin{array}{c}
s \\
n_{s}
\end{array}\right]}{\partial a(n ; \zeta)} \mu_{r}^{v^{\prime}+\bar{\gamma}} \prod_{p \neq n_{s}}\left(R_{\tilde{\varepsilon}}^{\gamma^{\prime}}\left[\begin{array}{c}
s \\
p
\end{array}\right] \mu_{r}^{v^{\prime}}\right),
$$

где $\tilde{\varepsilon}=\left(\varepsilon_{1}, \ldots, \varepsilon_{s-1}, \varepsilon_{s+1}, \ldots, \varepsilon_{N}\right), \gamma^{\prime}=\zeta_{s}, v=\delta_{1-2 \varepsilon_{j_{r}}}^{\bar{\gamma}} Q_{j_{r}}, v^{\prime}=\delta_{1-2 \varepsilon_{j_{r}}}^{\bar{\gamma}} Q_{j_{r}, j_{s}}$, $Q_{j_{r}, j_{s}}=Q_{j_{r}} / T_{j_{s}}^{\prime}$. Так как для общего $L$ справедливо включение $L \in U_{t r}$, то из (23) и (24) следует, что

$$
\left.\frac{\partial R_{\varepsilon^{\prime}}^{\gamma^{\prime}\left[\begin{array}{c}
s \\
n_{s}
\end{array}\right]}}{\partial a(n ; \zeta)} \mu_{r}^{v^{\prime}+\bar{\gamma}}\right|_{X_{\varepsilon^{\prime}}^{\gamma^{\prime}, \bar{\gamma}}\left[\begin{array}{c}
s, r \\
n_{s}, n_{r}
\end{array}\right]} \equiv 0,
$$

где $\varepsilon^{\prime}=\left(\varepsilon_{1}, \ldots, \varepsilon_{r-1}, \varepsilon_{r+1}, \ldots, \varepsilon_{s-1}, \varepsilon_{s+1}, \ldots, \varepsilon_{N}\right)$, а это противоречит предположению индукции. Тот факт, что $W_{R} \neq \varnothing$, доказывается аналогично.

Докажем, что $U_{s m} \neq \varnothing$. Действительно, полиномы $R_{\varepsilon}$ линейно зависят от коэффициентов $a(n, \zeta)$, т. е. $X_{\varepsilon}$ образуют линейную систему дивизоров на $\left(\mathbb{C P}^{1}\right)^{N}$. Теорема Бертини о линейных системах дивизоров утверждает, что общий член линейной системы не имеет особых точек вне базисного множества, т.е. если $z \in \operatorname{Sing} X_{\varepsilon}$ для общего $L$, то $z \in X_{\varepsilon}(L)$ для всех $L$. Таким образом, лемма 3 завершает доказательство того, что $U_{s m} \neq \varnothing$.

Докажем, что $U_{E} \neq \varnothing$, индукцией по $N$. Если $U_{E}=\varnothing$ (т. е. $\mathscr{E}_{\varepsilon} \neq 0$ для общего оператора $L_{\varepsilon}$ ), то из элементарной теории пересечения следует, что существуют $\gamma, t, u \in \mathbb{Z}$, такие, что $\mathscr{E}_{\varepsilon}^{\gamma}\left[\begin{array}{l}t \\ u\end{array}\right]=\mathscr{E} \cap X_{\varepsilon}^{\gamma}\left[\begin{array}{l}t \\ u\end{array}\right] \neq 0 ;$ следовательно, $U_{E}^{\gamma}\left[\begin{array}{l}t \\ u\end{array}\right]=\varnothing$, а это противоречит предположению индукции.

Если многообразие $X_{\varepsilon} \subset\left(\mathbb{C P}^{1}\right)^{N}$ приводимо и неособо, то $X_{\varepsilon}=X_{\varepsilon}^{\prime} \cup X_{\varepsilon}^{\prime \prime}$, $X_{\varepsilon}^{\prime} \cap X_{\varepsilon}^{\prime \prime}=\varnothing$, а следовательно, $X_{\varepsilon}^{\prime}$ и $X_{\varepsilon}^{\prime \prime}$ должны иметь степени $\left(a_{1}^{\prime}, \ldots, a_{N}^{\prime}\right)$, $\left(a_{1}^{\prime \prime}, \ldots, a_{N}^{\prime \prime}\right)$, такие, что $a_{r}^{\prime} a_{r}^{\prime \prime}=0, r=1, \ldots, N$. Точно так же, как и раньше, индукцией по размерности доказывается невозможность такого распадения для компонент модифицированного спектрального многообразия общего оператоpa $L$.

Таким образом, мы показали, что $\mathfrak{D}$ определено на плотном открытом подмножестве пространства $\mathfrak{L}_{T}$. Введем обозначение $\mathfrak{D}\left(\mathfrak{L}_{T}\right)=\mathfrak{M}_{T}^{\prime} \subset \mathfrak{M}_{T}$.

Теорема 2. Отображение $\mathfrak{D}: \mathfrak{L}_{T} \rightarrow \mathfrak{M}_{T}^{\prime}$ обратимо на некотором всюду плотном множестве. Отображение $\mathfrak{I}=\mathfrak{D}^{-1}$ может быть описано с помощью $\theta$-функций кривьцх $X_{\varepsilon}\left[\begin{array}{l}i \\ k\end{array}\right], i, k \in \mathbb{Z}^{N-2}$. 
ДоКАЗАТЕЛЬСтВО. В силу предложения 3 мы можем проводить построение отображения $\mathfrak{I}$ по индукции. Таким образом, предположим, что для размерностей меньше $N$ отображение $\mathfrak{I}$ уже построено, и, исходя из этого, построим отображение $\mathfrak{I}$ в размерности $N$.

Заметим, что для собственной функции $\psi, L \psi=0$, имеет место равенство

$$
a(n ;-1, \gamma) \psi^{\gamma}\left[\begin{array}{c}
2,3, \ldots, N \\
k_{1}, k_{2}, \ldots, k_{N-1}
\end{array}\right]\left(n_{1}-1\right)+a(n ; 1, \gamma) \psi^{\gamma}\left[\begin{array}{c}
2,3, \ldots, N \\
k_{1}, k_{2}, \ldots, k_{N-1}
\end{array}\right]\left(n_{1}+1\right)=0 .
$$

Выполнение этих равенств при всех $n \in \mathbb{Z}^{N}$ эквивалентно выполнению следующих равенств:

$$
\begin{gathered}
\frac{a(n ; 1, \gamma)}{a\left(n_{1}+2, \tilde{n} ; 1, \gamma\right)}=\frac{\psi^{-\gamma}\left[\begin{array}{c}
2, \ldots, N \\
k_{1}, \ldots, k_{N-1}
\end{array}\right]\left(n_{1}+2\right)}{\psi^{-\gamma}\left[\begin{array}{c}
2, \ldots, N \\
k_{1}, \ldots, k_{N-1}
\end{array}\right]\left(n_{1}\right)} \frac{\psi^{\gamma}\left[\begin{array}{c}
2, \ldots, N \\
k_{1}, \ldots, k_{N-1}
\end{array}\right]\left(n_{1}+3\right)}{\psi^{\gamma}\left[\begin{array}{c}
2, \ldots, N \\
k_{1}, \ldots, k_{N-1}
\end{array}\right]\left(n_{1}+1\right)}, \\
\frac{a(n ;-1, \gamma)}{a\left(n_{1}+2, \tilde{n} ;-1, \gamma\right)}=\frac{\psi^{\gamma}\left[\begin{array}{c}
2, \ldots, N \\
k_{1}, \ldots, k_{N-1}
\end{array}\right]\left(n_{1}+1\right)}{\psi^{\gamma}\left[\begin{array}{c}
2, \ldots, N \\
k_{1}, \ldots, k_{N-1}
\end{array}\right]\left(n_{1}-1\right)} \frac{\psi^{-\gamma}\left[\begin{array}{c}
2, \ldots, N \\
k_{1}, \ldots, k_{N-1}
\end{array}\right]\left(n_{1}+2\right)}{\psi^{-\gamma\left[\begin{array}{c}
2, \ldots, N \\
k_{1}, \ldots, k_{N-1}
\end{array}\right]\left(n_{1}\right)}}
\end{gathered}
$$

при всех $n \in \mathbb{Z}^{N}$; в этих формулах $\tilde{n}=\left(n_{2}, \ldots, n_{N}\right)$.

Теперь выберем мероморфные на $X_{\varepsilon}$ функции $\psi_{\varepsilon}(n)$ так, чтобы выполнялись равенства (11) и $\psi_{\varepsilon}(n)=\psi_{\varepsilon+e_{h_{r}}}\left(n+T_{h_{r}}^{\prime} e_{r}\right)$, где $\varepsilon_{h}=0, r=1, \ldots, N-S$, $n \in \Gamma_{1-\varepsilon}^{N}$, а $\psi(n)$ суть $T^{\prime}$-блоховские функции с множителями Флоке $\mu$. С помощью функций $\psi^{-1}\left[\begin{array}{l}1 \\ 0\end{array}\right], \psi^{-1}\left[\begin{array}{l}1 \\ 1\end{array}\right]$, определяемых формулой (14), по предположению индукции мы можем построить операторы $M_{0} \psi^{-1}\left[\begin{array}{l}1 \\ 0\end{array}\right]=0, M_{1} \psi^{-1}\left[\begin{array}{l}1 \\ 1\end{array}\right]=0$. Пусть коэффициенты оператора $M_{e}, e=0,1$, равны $a_{e}(k, \zeta), \zeta, k \in \mathbb{Z}^{N-1}$, $\zeta_{r}= \pm 1, r=1, \ldots, N-1$. Формулы $(25)$, (26) позволяют выразить коэффициенты $a(2 s+1, \tilde{n} ; \gamma)$ через коэффициенты $a(1, \tilde{n} ; \gamma)$. Таким образом, $(25),(26)$ вместе с равенством $a(1, k ; 1-2 e, \tilde{\gamma})=a_{e}(k ; \tilde{\gamma}), e=0,1$, дают нам отображение $\mathfrak{I}$, причем произвол в выборе $\psi$ отвечает действию калибровочной группы. Следовательно, $\mathfrak{I}$ является корректно определенным отображением из $\mathfrak{M}_{T}$ в $\mathfrak{L}_{T}$ и по построению $\mathfrak{I D}=\mathrm{id}$.

Заметим, что дроби, присутствующие в формулах (25), (26), могут быть выражены в терминах $\theta$-функций кривых $X_{\varepsilon}\left[\begin{array}{c}3,4, \ldots, N \\ k_{1}, k_{2}, \ldots, k_{N-2}\end{array}\right]$ (см. [6]).

Отметим, что доказательство теоремы 2 дает нам индуктивный способ построения I. В частности, при $N=3$ и $h=\varnothing$ (т. е. $S=3$ ) процедура решения обратной спектральной задачи выглядит так. Сначала необходимо пересечь $\mathscr{D}_{\varepsilon}$, $\varepsilon_{i}=0,1, i=1,2,3$, с кривыми $X_{\tilde{\varepsilon}}^{-1}\left[\begin{array}{c}3 \\ 2-\varepsilon_{3}+2 t\end{array}\right], 1 \leqslant t \leqslant T_{i} / 2$. В результате получим дивизоры $\mathscr{F}_{\tilde{\varepsilon}}(t)=X_{\tilde{\varepsilon}}^{-1}\left[\begin{array}{c}3 \\ 2-\varepsilon_{3}+2 t\end{array}\right] \cap \mathscr{D}_{\varepsilon}$. Пересекая левую часть формулы $(19)$ с $X_{\tilde{\varepsilon}}^{-1}\left[\begin{array}{c}3 \\ 2-\varepsilon_{3}+2 t\end{array}\right]$ при $n^{0}=1-\varepsilon+2 t e_{3}, n^{1}=1-\varepsilon$ и учитывая (16) и (15), получаем связь между $\mathscr{F}_{\tilde{\varepsilon}}^{\gamma}(t)$ и $\mathscr{D}_{1-\tilde{\varepsilon}}^{-1}\left[\begin{array}{c}3 \\ 2-\varepsilon_{3}+2 t\end{array}\right]$, которая имеет вид $\mathscr{D}_{1-\tilde{\varepsilon}}^{-1}\left[\begin{array}{c}3 \\ 2-\varepsilon_{3}+2 t\end{array}\right] \sim$ $\mathscr{F}_{\tilde{\varepsilon}}(t)-t\left(T_{1} \sum_{i=1}^{T_{2}} X_{\varepsilon_{1}}^{-1,1}\left[\begin{array}{c}3,2 \\ 2-\varepsilon_{3}+2 t, \varepsilon_{2}+2 i\end{array}\right]+T_{2} \sum_{i=1}^{T_{1}} X_{\varepsilon_{2}}^{-1,1}\left[\begin{array}{c}3,1 \\ 2-\varepsilon-3+2 t, \varepsilon_{1}+2 i\end{array}\right]\right)$. С помощью формулы (16) по дивизорам $\mathscr{D}_{1-\tilde{\varepsilon}}^{-1}\left[\begin{array}{c}3 \\ 2-\varepsilon_{3}+2 t\end{array}\right]$ строим дивизоры $\mathscr{D}_{\tilde{\varepsilon}}^{1}\left[\begin{array}{c}3 \\ 1-\varepsilon_{3}+2 t\end{array}\right]$.

Согласно лемме $2, \operatorname{dim} H^{0}\left(X_{\tilde{\varepsilon}}^{\gamma}\left[\begin{array}{c}3 \\ \varepsilon_{3}+2 t\end{array}\right], \mathscr{O}\left(\mathscr{D}_{1-\varepsilon}^{\gamma}+Q^{\gamma}\left[\begin{array}{c}3 \\ \varepsilon_{3}+2 t\end{array}\right]\left[\begin{array}{c}m^{1} \\ m^{0}\end{array}\right]\right)\right)=1, m^{0}, m^{1} \in$ $\Gamma_{1-\tilde{\varepsilon}}^{2}$; выберем функции $\psi_{\tilde{\varepsilon}}^{\gamma}\left[\begin{array}{c}3 \\ \varepsilon_{3}+2 t\end{array}\right] \in H^{0}\left(X_{\tilde{\varepsilon}}^{\gamma}\left[\begin{array}{c}3 \\ \varepsilon_{3}+2 t\end{array}\right], \mathscr{O}\left(\mathscr{D}_{1-\varepsilon}^{\gamma}+Q^{\gamma}\left[\begin{array}{c}3 \\ \varepsilon_{3}+2 t\end{array}\right]\left[\begin{array}{c}m^{1} \\ m^{0}\end{array}\right]\right)\right)$. Произвол в выборе этих функций отвечает действию калибровочной группы. По 
определению оператор $L_{\tilde{\varepsilon}}^{\gamma}\left[\begin{array}{c}3 \\ \varepsilon_{3}+2 t\end{array}\right]$ действует из пространства $V_{1-\tilde{\varepsilon}}^{2}$ в $V_{\tilde{\varepsilon}}^{2}$, а оператор $L_{1-\tilde{\varepsilon}\left[\varepsilon_{3}+\gamma+2 t\right.}^{-\gamma}$ - из $V_{\tilde{\varepsilon}}^{2}$ в $V_{1-\tilde{\varepsilon}}^{2}$, и, согласно формуле $(5)$, эти операторы сопряжены друг другу. Эти два оператора следует рассматривать как две части одного самосопряженного оператора $\widehat{L}_{\tilde{\varepsilon}}^{\gamma}\left[\begin{array}{c}3 \\ \varepsilon_{3}+2 t\end{array}\right]$, действующего на пространстве $V_{\tilde{\varepsilon}}^{2} \oplus V_{1-\tilde{\varepsilon}}^{2}$ по формуле $\left(\widehat{L}_{\tilde{\varepsilon}}^{\gamma}\left[\begin{array}{c}3 \\ \varepsilon_{3}+2 t\end{array}\right] \psi\right)(m)=\left(L_{\tilde{\varepsilon}}^{\gamma}\left[\begin{array}{c}3 \\ \varepsilon_{3}+2 t\end{array}\right] \psi\right)(m)$, если $m \in \Gamma_{1-\tilde{\varepsilon}}$, и по формуле $\left(\widehat{L}_{\tilde{\varepsilon}}^{\gamma}\left[\begin{array}{c}3 \\ \varepsilon_{3}+2 t\end{array}\right] \psi\right)(m)=\left(L_{1-\tilde{\varepsilon}}^{-\gamma}\left[\begin{array}{c}3 \\ \varepsilon_{3}+2 t+\gamma\end{array}\right] \psi\right)(m)$, если $m \in \Gamma_{\tilde{\varepsilon}}$. Заметим также, что $\widehat{L}_{\tilde{\varepsilon}}^{\gamma}\left[\begin{array}{c}3 \\ \varepsilon_{3}+2 t\end{array}\right]=\widehat{L}_{1-\tilde{\varepsilon}}^{-\gamma}\left[\begin{array}{c}3 \\ \varepsilon_{3}+2 t+\gamma\end{array}\right]$ и инволюция $\sigma$ отождествляет кривые $X_{\tilde{\varepsilon}}^{\gamma}\left[\begin{array}{c}3 \\ \varepsilon_{3}+2 t\end{array}\right]$ и $X_{1-\tilde{\varepsilon}\left[\varepsilon_{3}+2 t+\gamma\right.}^{-\gamma}$. Из результатов работы [6] следует, что существует единственный оператор $\widehat{L}_{\tilde{\varepsilon}}^{\gamma}\left[\begin{array}{c}3 \\ \varepsilon_{3}+2 t\end{array}\right]$, такой, что $\widehat{L}_{\tilde{\varepsilon}}^{\gamma}\left[\begin{array}{c}3 \\ \varepsilon_{3}+2 t\end{array}\right] \hat{\psi}_{\tilde{\varepsilon}}^{\gamma}\left[\begin{array}{c}3 \\ \varepsilon_{3}+2 t\end{array}\right]=0$, где $\hat{\psi}_{\tilde{\varepsilon}}^{\gamma}\left[\begin{array}{c}3 \\ \varepsilon_{3}+2 t\end{array}\right](m)=$ $\psi_{\tilde{\varepsilon}}^{\gamma}\left[\begin{array}{c}3 \\ \varepsilon_{3}+2 t\end{array}\right](m)$, если $m \in \Gamma_{1-\tilde{\varepsilon}}^{2}$, и $\hat{\psi}_{\tilde{\varepsilon}}^{\gamma}\left[\begin{array}{c}3 \\ \varepsilon_{3}+2 t\end{array}\right](m)=\psi_{1-\tilde{\varepsilon}}^{-\gamma}\left[\begin{array}{c}3 \\ \varepsilon_{3}+2 t+\gamma\end{array}\right](m)$, если $m \in \Gamma_{\tilde{\varepsilon}}^{2}$. Коэффициенты оператора $\widehat{L}_{\tilde{\varepsilon}}^{\gamma}\left[\begin{array}{c}3 \\ \varepsilon_{3}+2 t\end{array}\right]$ выписываются в терминах $\theta$-функций кривой $X_{\tilde{\varepsilon}}^{\gamma}\left[\begin{array}{c}3 \\ \varepsilon_{3}+2 t\end{array}\right]$. Теперь нам осталось устранить произвол в выборе константы, с точностью до умножения на которую определен $\widehat{L}_{\tilde{\varepsilon}}^{\gamma}\left[\begin{array}{c}3 \\ \varepsilon_{3}+2 t\end{array}\right]$. Это можно сделать, потребовав выполнения равенств $(25),(26)$ (для $N=3$ ). После этого оператор $L$ определен функциями $\psi_{\tilde{\varepsilon}}^{\gamma}\left[\begin{array}{c}3 \\ \varepsilon_{3}+2 t\end{array}\right]$ однозначно с точностью до умножения на константу. Следовательно, мы восстановили оператор $L$ из спектральных данных $\left\{X_{\varepsilon}, \mathscr{D}_{\varepsilon}\right\}$.

\section{ЛИТЕРАТУРА}

1. Шадан K., Сабатье П. Обратные задачи в квантовой теории рассеяния. Мир, М., 1980.

2. Дубровин А. Б., Кричевер И. М., Новиков С. П. Уравнение Шрёдингера в периодическом поле и римановы поверхности. ДАН СССР, 229, № 1, 15-18 (1976).

3. Веселов А. П., Новиков С. П. Конечнозонные двумерные операторы Шрёдингера: явные формулы и эволюционные уравнения. ДАН СССР, 279, № 1, 20-24 (1984).

4. Веселов А. П., Новиков С. П. Конечнозонные двумерные операторы Шрёдингера. Потенциальные операторы, ДАН СССР, 279, № 4, 784-788 (1984).

5. Novikov S. P., Veselov A. P. Two-dimensional Schrodinger operator: inverse scattering problem and evolutional equations. Phys. D, 18, 267-273 (1986).

6. Кричевер И. М. Двумерные периодические разностные операторы и алгебраическая геометрия. ДАН СССР, 285, № 1, 31-36 (1985).

7. Veselov A. P., Krichever I. M., Novikov S. P. Two-dimensional periodic Schrodinger operators and Prym's $\theta$-functions. Progress in math, Vol. 60, Geometry today, 1985, pp. 283-301.

8. Обломков A. A. О разностных операторах на двумерных правильных решетках. Теор. матем. физика, 127, № 1, 34-46 (2001).

9. Oblomkov A. A., Penskoi A. V. Two-dimensional algebro-geometric operators. J. Phys. A: Math. Gen., 33, 9255-9264 (2000).

10. Kappeler T. On isospectral potentials on discrete lattice II. Adv. Appl. Math., 9, No. 4, 428-438 (1988).

11. Bättig D. A. A toroidal compactification of the complex Fermi surface. Comment. Math. Helv., 65, No. 1, 144-149 (1990).

12. Knörrer H., Trubowitz E. A directional compactification of the complex Boch variety. Comment. Math. Helv., 69, No. 1, 144-149 (1990).

13. Bättig D., Knörrer H., Trubowitz E. A directional compactification of the complex Fermi surface. Compositio Math., 79, No. 2, 205-229 (1991). 
14. Новиков С. П., Дьнников И. А. Дискретные спектральные симметрии маломерных дифференциальных операторов и разностные операторы на правильных решетках и двумерных многообразиях. УМН, 52, вып. 5, 175-234 (1997).

15. Новиков С. П., Дынников И. А. Преобразования Лапласа и симплициальные связанности. УМН, 52, вып. 6, 157-158 (1997).

16. Обломков A. A. О спектральных свойствах двух классов разностных периодических операторов. Матем. сб., 193, № 4, 87-112 (2002).

17. Дубровин Б. А., Матвеев В. Б., Новиков С. П. Нелинейные уравнения типа Кортевега-де Фриза, конечнозонные линейные операторы и абелевы многообразия. УМН, 31, вып. 1, 55-136 (1976).

18. Веселов А. П. Интегрируемые системы с дискретным временем и разностные операторы. Функц. анализ и его прил., 22, вып. 2, 1-13 (1988).

19. Гриффитс Ф., Харрис Дж. Принципы алгебраической геометрии. В 2-х томах. Мир, M., 1982.

Московский государственный

университет им. М. В. Ломоносова,

Поступило в редакцию

Независимый московский университет

27 ноября 2000 г.

e-mail: oblomkov@math.mit.edu 